\title{
On the Long-Term Fitness of Cells in Periodically Switching Environments
}

\author{
Ning-Ning Pang ${ }^{\mathrm{a}, *}$, Wen-Jer Tzeng ${ }^{\mathrm{b}, *}$ \\ ${ }^{a}$ Department of Physics, National Taiwan University, Taipei, Taiwan, ROC \\ ${ }^{b}$ Department of Physics, Tamkang University, Tamsui, Taipei, Taiwan, ROC
}

Received: 31 March 2006 / Accepted: 22 June 2007 / Published online: 18 August 2007

(C) Society for Mathematical Biology 2007

\begin{abstract}
Because all the cell populations are capable of making switches between different genetic expression states in response to the environmental change, Thattai and van Oudenaarden (Genetics 167, 523-530, 2004) have raised a very interesting question: In a constantly fluctuating environment, which type of cell population (heterogeneous or homogeneous) is fitter in the long term? This problem is very important to development and evolution biology. We thus take an extensive analysis about how the cell population evolves in a periodically switching environment either with symmetrical time-span or asymmetrical time-span. A complete picture of the phase diagrams for both cases is obtained. Furthermore, we find that the systems with time-dependent cellular transitions all collapse to the same set of dynamical equations with the modified parameters. Furthermore, we also explain in detail how the fitness problem bears much resemblance to the phenomenon, stochastic resonance, in physical sciences. Our results could be helpful for the biologists to design artificial evolution experiments and unveil the mystery of development and evolution.
\end{abstract}

Keywords Fitness · Biological switch · Population biology · Evolution · Bifurcation

\section{Introduction}

All the biological organisms contain global genetic regulatory networks, which consist of DNA transcription, RNA translation, RNA degradation, protein degradation, and modification of protein activity. Thus, the biological organisms are capable of making switches between different genetic expression states (Cherry and Adler, 2000) to fit their living environments. Among all the organisms on the earth, bacteria are ubiquitous because of their extraordinary ability to sense and promptly respond to fast changes of the environmental conditions (including $\mathrm{pH}$, temperature, osmolarity, nutrients, and others). Usually,

\footnotetext{
*Corresponding authors.

E-mail addresses: nnp@phys.ntu.edu.tw (Ning-Ning Pang), wjtzeng @ mail.tku.edu.tw (Wen-Jer Tzeng).
} 
bacteria sense and respond to changes in the environment through a network of two component signal transduction mechanisms. Specifically, a sensor/kinase component spanning the cell membrane senses an environmental signal and then transmits the signal to a regulatory protein located in the cytoplasm through phosphorylation/dephosphorylation reactions. The phosphorylated regulatory protein then regulates target gene expression.

For illustration, we list a few paradigm examples in bacterial genetic switch in response to the environmental change. The first example is the lac operon of bacterium $E$. coli (Schlax et al., 1995). An operon is a few genes situated in sequence and controlled by a common regulatory region. If the glucose supply from the environment is lacking, the lac operon will be expressed for the utilization of lactose as a carbon source. The second example is bacterial flagellar phase variation switch (Macnab, 1992). Bacterial flagella are long hollow cylindrical filaments that extend from the bacterial cell surface. The basal body of flagellum contains a motor, which propels the cell to move through an aqueous environment. The bacterium S. enterica can alter the forms of their flagellin proteins to avoid the attack of mammalian immune systems. Usually, the bacteria switch the flagellar gene expression at frequencies from $10^{-3}$ to $10^{-5}$ per cell per generation. The next example is aerobic to anaerobic transitions for facultative bacteria (Unden et al., 1995). Facultative bacteria are capable of using either respiratory or fermentation processes to accommodate growth and multiplication, depending on the availability of oxygen in the environment. Facultative bacteria execute this switch through the alteration of electron flow pathway in the metabolism. The last example is Pap pili and type 1 pili phase variation in bacteria (Hernday et al., 2002). Pili are shorter and more rigid hairs (than flagella) on the bacterial cell surface. The bacterium uses pili to adhere on host cells. Uropathogenic E. coli expresses its type 1 pili or Pap pili to colonize the lower or upper, respectively, urinary tract of humans. Pili phase variation can be stimulated by a variety of environmental factors, such as temperature, $\mathrm{pH}$, and medium richness. The experimental data suggest that expression of Pap pili or type 1 pili is mutually exclusive.

Note that all the biochemical reactions are intrinsically stochastic (Spudich and Koshland, 1976; Paulsson, 2004; Wolf and Arkin, 2002; Kepler and Elston, 2001; Gardner et al., 2000). Low numbers of molecules in each cell make the random fluctuations distinct. In addition, temperature fluctuation and other variation of physical or chemical conditions affect chemical reaction rates and may influence the qualitative system behaviors. Thus, it is generally believed that how to regulate noise is one of the main features of any design of biochemical networks in the living systems. For example, living systems can use negative feedback, integral feedback, parallel cascades, regulatory checkpoints, and even proofreading in protein translation to attenuate the noise (Becskei and Serrano, 2000; Barkai and Leibler, 1997; Warren and ten Wolde, 2004; Murray, 2002; Rao et al., 2002). However, is the noise such a nuisance? Since all the biological organisms have been always evolving in such a noisy environment, they should have evolved the ability to exploit the noise for self-optimization instead of just passively avoiding it. Remarkably, Leibler and colleagues (Vilar et al., 2002) studied the mechanism of circadian clocks and surprisingly found, in some situations, the addition of suitable noise can stimulate regular biological oscillations when the original deterministic models fail to do so. Interestingly, in the recent 20 years, scientists have noticed a peculiar phenomenon: An appropriate dosage of noise can actually enhance the performance of many technological devices. This phenomenon has coined 
the term "stochastic resonance" in physics and engineering (Gammaitoni et al., 1998; Moss, 1994).

Contemporary works begin to focus on cellular processes which exploit noise (Rao et al., 2002). Although the particular phase variation of each cell seems random individually, regulatory factors tune mean levels of population heterogeneity. Does population heterogeneity help or hurt development and evolution of living things? Since cells are able to switch between different genetic expression states, Thattai and van Oudenaarden (2004) raise a very important question: In a constantly fluctuating environment, which type of cell population (heterogeneous or homogeneous) is fitter in the long term? In their paper, they also proposed a simple but generic dynamical model to qualitatively formulate the problem. This question is one of the most interesting problems in development and evolutionary biology. Indeed, heterogeneity seems to help survival of population at least in some known biological cases. For example, the fim network of uropathogenic E. coli both exploits and filters noise to create appropriate heterogeneity in the cell population for long term survival (Hernday et al., 2002). It seems that population heterogeneity spreads risk over multiple phenotypes. But is it always a best strategy? Furthermore, recent genetic experimental results support the concept of adaptive mutation in response to the environment (Rosenberg, 2001; Tanaka et al., 2003). How do biological organisms deal with the conflicting needs for robustness and flexibility in the face of uncertainty? What is its link to stochastic resonance? Can we borrow the concept of stochastic resonance to obtain a better understanding of pathway evolution for the use of cellular bioengineering in the future?

Since these problems are very important in evolutionary biology and artificial evolution experiments, we are strongly motivated to take a detailed investigation about how the cell population evolves in a periodically switching environment. In this paper, we plan to undertake extensive analytical and numerical studies on the overall population fitness in a periodically switching environment either with symmetrical time-span or asymmetrical time-span. After obtaining the whole phase diagrams, a comprehensive mathematical, physical, and biological reasoning about the various asymptotes in the phase diagrams will be given. Moreover, we also consider the switching dynamics of cell populations with the effect of cellular transition time explicitly taken into account. Furthermore, we will discuss in detail how the problem of optimal fitness in switching environments bears much resemblance to a renowned phenomenon, stochastic resonance, in physics and engineering. Hence, our analysis will be helpful for the biologists to design the evolution experiments, measure population heterogeneity under varying conditions, and pin down the network evolution theory in the future.

\section{Periodically switching environments with symmetrical time-span}

\subsection{Basic properties}

First, we will start with a recently proposed model (Thattai and van Oudenaarden, 2004), a minimal but generic dynamical model, mimicking the biological switch of cells in changing environments. The biological switches enable cells to respond to environmental signals. This simple model, as in any general model, is not intended for the specific details but to capture the essential core properties. The definition of the model is as follows. The 
environment can switch between two environmental states $e_{a}$ and $e_{b}$ with equal time-span in each state. Each cell in the population allows two distinct cell states $c_{a}$ and $c_{b}$. In environmental state $e_{a}$, cell state $c_{a}$ is the fit state with a larger growth rate per capita and cell state $c_{b}$ is the unfit state with a smaller growth rate per capita; when the environment flips to the state $e_{b}$, cell state $c_{b}$ is the fit state and cell state $c_{a}$ is the unfit state. Since we are focusing on the issue about the long term fitness of the cell population, under either environmental condition the fit (unfit) state of the cells is labeled by $c_{1}\left(c_{0}\right)$ with the growth rate per capita $\gamma_{1}\left(\gamma_{0}\right)$. Set $\Delta \gamma \equiv \gamma_{1}-\gamma_{0}>0$, consistent with the fact that $c_{1}$ is the fit state. All the biochemical reactions are intrinsically stochastic. The stochastic transitions between these two cell states are described by the switching rate coefficients $k_{1}$ from $c_{0}$ to $c_{1}$ and $k_{0}$ in the reverse direction. Thus, the dynamical equations, constructed in terms of the number of cells $n_{1}$ in the fit state and the number of cells $n_{0}$ in the unfit state, are given by

$$
\begin{aligned}
& \frac{d n_{0}}{d t}=\gamma_{0} n_{0}-k_{1} n_{0}+k_{0} n_{1}, \\
& \frac{d n_{1}}{d t}=\gamma_{1} n_{1}-k_{0} n_{1}+k_{1} n_{0} .
\end{aligned}
$$

We are interested in the fraction of cells in the fit state, $f_{1}(t) \equiv n_{1} /\left(n_{1}+n_{0}\right)$. Then, the dynamics of $f_{1}$ is described by

$$
\begin{aligned}
\frac{d f_{1}}{d t} & =\frac{1}{\left(n_{1}+n_{0}\right)} \frac{d n_{1}}{d t}-\frac{n_{1}}{\left(n_{1}+n_{0}\right)^{2}} \frac{d\left(n_{1}+n_{0}\right)}{d t} \\
& =k_{1}+\left(\Delta \gamma-k_{0}-k_{1}\right) f_{1}-\Delta \gamma f_{1}^{2},
\end{aligned}
$$

under the condition of the two-state switching environment. Suppose that the environment spends a fixed duration of time $T$ in each state and, thus, Eq. (3) needs to be regulated by the condition $f_{1}\left(t=(n T)^{-}\right)=f_{0}\left(t=(n T)^{+}\right)=1-f_{1}\left(t=(n T)^{+}\right)$.

The dynamical equation of $f_{1}$, Eq. (3), is exactly solvable either without or with the constraints imposed by the switching environment. Let us first analyze its steady state solution and the overall qualitative behavior. The steady state solution $\tilde{f}_{1}$ is given by $k_{1}+\left(\Delta \gamma-k_{0}-k_{1}\right) \tilde{f}_{1}-\Delta \gamma \tilde{f}_{1}^{2}=0$, leading to

$$
\tilde{f}_{1}=\frac{\Delta \gamma-k_{0}-k_{1} \pm\left[\left(\Delta \gamma+k_{0}+k_{1}\right)^{2}-4 \Delta \gamma k_{0}\right]^{1 / 2}}{2 \Delta \gamma} \equiv \tilde{f}_{ \pm} .
$$

The negative solution $\tilde{f}_{-}<0$ is not an acceptable physical solution. From the inequality $\left|\Delta \gamma-k_{0}-k_{1}\right| \leq\left[\left(\Delta \gamma+k_{0}+k_{1}\right)^{2}-4 \Delta \gamma k_{0}\right]^{1 / 2} \leq \Delta \gamma+k_{0}+k_{1}$, we always have $0 \leq$ $\tilde{f}_{+} \leq 1$. To be biologically reasonable, we further require $0 \leq k_{0}<k_{1}+\Delta \gamma / 2$, and thus have $1 / 2<\tilde{f}_{+} \leq 1$ as expected. Equation (3), the rate equation for $f_{1}$, can be recast as $d f_{1} / d t=\Delta \gamma\left(\tilde{f}_{+}-f_{1}\right)\left(f_{1}-\tilde{f}_{-}\right)$. With some calculation, we have

$$
f_{1}(t)=\tilde{f}_{+}-\frac{\tilde{f}_{+}-\tilde{f}_{-}}{1+A \rho^{t}}
$$

with $A \equiv\left[f_{1}(0)-\tilde{f}_{-}\right] /\left[\tilde{f}_{+}-f_{1}(0)\right]$ and $\rho \equiv \exp \left[\Delta \gamma\left(\tilde{f}_{+}-\tilde{f}_{-}\right)\right]$. For $f_{1}(0)<\tilde{f}_{+}, A>0$ and $f_{1}(t) \nearrow \tilde{f}_{+}$as $t$ increases. For $f_{1}(0)>\tilde{f}_{+}, A<0$ and $f_{1}(t) \searrow \tilde{f}_{+}$as $t$ increases. So 

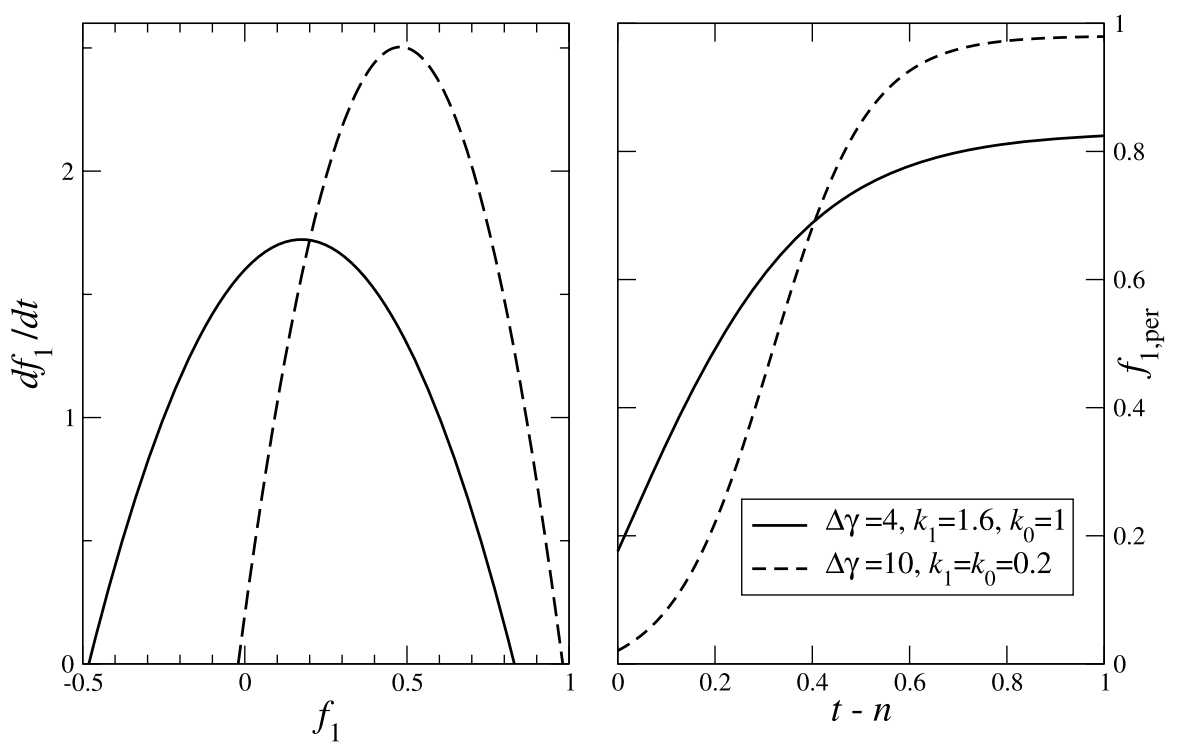

Fig. 1 (Left) A plot of $\left(d f_{1} / d t\right)$ vs $f_{1}$ in the range of $\left[\tilde{f}_{-}, \tilde{f}_{+}\right]$. Note that $0 \leq f_{1} \leq 1$ and $\tilde{f}_{-} \leq 0$ for any value of $\left(k_{0}, k_{1}, \Delta \gamma\right)$. In contrast, $1 / 2<\tilde{f}_{+} \leq 1$ for $0 \leq k_{0}<k_{1}+\Delta \gamma / 2$. (Right) For cell populations in periodically switching environments with symmetrical time-span, we plot an illustrative figure of $f_{1 \text {,per }}(t)$ vs $t-n$ with $n<t<n+1$ for $f_{1, \text { per }}\left(t=n^{+}\right)>\left(\tilde{f}_{+}+\tilde{f}_{-}\right) / 2=1 / 2-\left(k_{0}+k_{1}\right) /(2 \Delta \gamma)$ (solid curve) and $<\left(\tilde{f}_{+}+\tilde{f}_{-}\right) / 2$ (dashed curve).

we always have $f_{1}(t) \rightarrow \tilde{f}_{+}$in the time intervals between two consecutive environmental changes.

Let us now impose the condition of the two-state switching environment. The environment periodically switches between two states, spending a fixed duration of time $T$ in each state. Without losing generality, we can set $T=1$. Thus, $f_{1}\left(t=n^{+}\right)=$ $1-f_{1}\left(t=n^{-}\right)$for any integer $n$. After some transient time, $f_{1}$ approaches its periodic state, i.e., $f_{1, \text { per }}(t)=f_{1 \text {, per }}(t+1)$. The qualitative behavior of $f_{1}(t)$ in the periodic state can be described as follows: $0<f_{1, \text { per }}\left(n^{+}\right)<1 / 2$ and $f_{1 \text {,per }}(t)$ increases with $t$ until $f_{1, \text { per }}\left[(n+1)^{-}\right]=f_{1, \text { per }}\left(n^{-}\right)=1-f_{1, \text { per }}\left(n^{+}\right)>1 / 2$. Figure 1 illustrates this qualitative behavior. Note that for $f_{1 \text {,per }}\left(t=n^{+}\right)<\left(\tilde{f}_{+}+\tilde{f}_{-}\right) / 2$, the curve $f_{1 \text {, per }}(t)$ versus $t-n$ (with $n<t<n+1)$ has an inversion point. In contrast, for $f_{1, \text { per }}\left(t=n^{+}\right)>\left(\tilde{f}_{+}+\tilde{f}_{-}\right) / 2$, the curve is concave and the values of $f_{1 \text {,per }}\left(n^{+}\right)$and $f_{1, \text { per }}\left(n^{-}\right)$are closer to $1 / 2$ than those in the former case. Mathematically, these qualitative behaviors are key elements in determining which type of cell population (heterogeneous or homogeneous) is fitter in the long term. We will discuss this point later.

The exact solution of $f_{1, \text { per }}(t)$ can also be obtained. By employing three relations (Eq. (5), $f_{1}\left(t=n^{+}\right)=1-f_{1}\left(t=n^{-}\right)$, and $\left.f_{1 \text {,per }}(t)=f_{1 \text {,per }}(t+1)\right)$ and denoting the value of $f_{1, \text { per }}\left(n^{-}\right)$by $x$, we have the physically acceptable solution:

$$
x=\frac{1}{2}+\frac{\tilde{f}_{+}-\tilde{f}_{-}}{2} \frac{\rho+1}{\rho-1}
$$




$$
-\frac{1}{2}\left[\left(\tilde{f}_{+}-\tilde{f}_{-}\right)^{2}\left(\frac{\rho+1}{\rho-1}\right)^{2}-\left(\frac{2 k_{1}}{\Delta \gamma}-\frac{2 k_{0}}{\Delta \gamma}+1\right)\right]^{1 / 2} .
$$

Consequently, the exact solution of the periodic state of $f_{1}(t)$ in the two-state switching environment with symmetrical time-span is obtained: $f_{1, \text { per }}(t)=\tilde{f}_{+}-\left(\tilde{f}_{+}-\tilde{f}_{-}\right) /(1+$ $\left.A_{\text {per }} \rho^{t-n}\right)$ with $A_{\text {per }}=\left(1-x-\tilde{f}_{-}\right) /\left(\tilde{f}_{+}-1+x\right)$, for $n<t<n+1$ and $n$ being an arbitrary integer.

In the following, we will show that the above solution of $f_{1 \text {,per }}(t)$ is an attractor. The time evolution of $f_{1}(t)$ can be described by the consecutive application of two maps. The first one is the flip map $\mathcal{F}$, operating at the moment when the environmental state switches; i.e., $\mathcal{F}\left(f_{1}\right)=1-f_{1}$. The other map $\mathcal{C}$ describes the changes of $f_{1}(t)$ in a given environmental state with the time duration equal to 1 ; i.e.,

$$
\mathcal{C}\left(f_{1}\right)=\tilde{f}_{+}-\frac{\tilde{f}_{+}-\tilde{f}_{-}}{1+\left(\frac{f_{1}-\tilde{f}_{-}}{\tilde{f}_{+}-f_{1}}\right) \rho} .
$$

Note that the quantity $x$ represents the instantaneous value of $f_{1, \text { per }}(t)$ just before the switching of the environmental states. Thus, the criterion for the stability of the periodic solution is that the absolute value of $\frac{d}{d x}[\mathcal{C} \circ \mathcal{F}(x)]$ is less than 1 . From $\left|\frac{d}{d x}[\mathcal{C} \circ \mathcal{F}(x)]\right|=$ $\left|\frac{\left(\tilde{f}_{+}-x\right)\left(x-\tilde{f}_{-}\right)}{\left(\tilde{f}_{+}-1+x\right)\left(1-x-\tilde{f}_{-}\right)}\right|<1$, we show that the periodic solution $f_{1, \mathrm{per}}(t)$ is an attractor of $f_{1}(t)$.

\subsection{The overall population fitness}

The mean growth rate per capita over the whole population is given by $\gamma=\left(\gamma_{1} n_{1}+\right.$ $\left.\gamma_{0} n_{0}\right) /\left(n_{1}+n_{0}\right)$. The time average of the mean growth rate per capita is thus $\langle\gamma\rangle_{t}=$ $\gamma_{0}+\Delta \gamma\left\langle f_{1}\right\rangle_{t} \equiv \gamma_{0}+\Delta \gamma f$, with $f \equiv\left\langle f_{1}\right\rangle_{t}$ denoting the time average of the fraction of cells in the fit state. As the value of $f$ is varied between zero and one, the time average of the mean growth rate per capita varies between $\gamma_{0}$ and $\gamma_{1} . f$ is thus the natural measure of the overall population fitness. Since we have obtained the exact solution of $f_{1 \text {,per }}(t)$, the explicit expression of $f$ in the periodically changing environment with symmetrical time-span is obtained as

$$
\begin{aligned}
f & =\int_{0}^{1} f_{1, \mathrm{per}}(t) d t=\int_{1-x}^{x} f_{1} \frac{d t}{d f_{1}} d f_{1} \\
& =\frac{\tilde{f}_{+}+\tilde{f}_{-}}{2}+\frac{1}{2 \Delta \gamma} \ln \frac{\left(\tilde{f}_{+}-1+x\right)\left(1-x-\tilde{f}_{-}\right)}{\left(\tilde{f}_{+}-x\right)\left(x-\tilde{f}_{-}\right)} .
\end{aligned}
$$

The main issue we want to explore is that in the two-state switching environment, what is the optimum strategy for the cell population to gain the long term fitness? In the following subsection, we will first solve the relation $\frac{\partial f}{\partial k_{0}}=0$ to get the optimal value of $k_{0}$ (i.e., $k_{0, \text { opt }}$ in short) as a function of $\Delta \gamma$ and $k_{1}$. Note that, under fixed environmental conditions, the cell population with $k_{1}>0$ and $k_{0}=0$ will eventually become homogeneous, while one with $k_{1}>0$ and $k_{0}>0$ will remain heterogeneous. Hence, the terms "heterogeneity" and "homogeneity" in this paper quantitatively refer to the situations with $k_{0, \mathrm{opt}}>0$ and $=0$, respectively. Then, we will analytically derive the various asymptotes in the phase space. Subsequently, a numerical investigation will be undertaken and the implications of the phase diagrams will be discussed. 


\subsubsection{Symbolic analysis of asymptotes}

First, we will solve the relation $\frac{\partial f}{\partial k_{0}}=0$ to get the relation among $\Delta \gamma, k_{1}$, and $k_{0, \text { opt }}$. From Eq. (7), we have

$$
\begin{aligned}
2 \Delta \gamma \frac{\partial f}{\partial k_{0}}+1= & \frac{\partial \tilde{f}_{+}}{\partial k_{0}}\left[\frac{1-2 x}{\left(\tilde{f}_{+}-1+x\right)\left(\tilde{f}_{+}-x\right)}\right]+\frac{\partial \tilde{f}_{-}}{\partial k_{0}}\left[\frac{1-2 x}{\left(1-x-\tilde{f}_{-}\right)\left(x-\tilde{f}_{-}\right)}\right] \\
& +\frac{\partial x}{\partial k_{0}}\left[\frac{2 \tilde{f}_{+}-1}{\left(\tilde{f}_{+}-1+x\right)\left(\tilde{f}_{+}-x\right)}-\frac{1-2 \tilde{f}_{-}}{\left(1-x-\tilde{f}_{-}\right)\left(x-\tilde{f}_{-}\right)}\right] .
\end{aligned}
$$

Let us express $\partial x / \partial k_{0}$ in terms of $\partial \tilde{f}_{+} / \partial k_{0}$ and $\partial \tilde{f}_{-} / \partial k_{0}$ :

$$
\begin{aligned}
\frac{\partial x}{\partial k_{0}} & {\left[\frac{2 \tilde{f}_{+}-1}{\left(\tilde{f}_{+}-1+x\right)\left(\tilde{f}_{+}-x\right)}+\frac{1-2 \tilde{f}_{-}}{\left(1-x-\tilde{f}_{-}\right)\left(x-\tilde{f}_{-}\right)}\right] } \\
= & \frac{\partial \tilde{f}_{+}}{\partial k_{0}}\left[\frac{2 x-1}{\left(\tilde{f}_{+}-1+x\right)\left(\tilde{f}_{+}-x\right)}+\Delta \gamma\right] \\
& -\frac{\partial \tilde{f}_{-}}{\partial k_{0}}\left[\frac{2 x-1}{\left(1-x-\tilde{f}_{-}\right)\left(x-\tilde{f}_{-}\right)}+\Delta \gamma\right] .
\end{aligned}
$$

By substituting Eqs. (4) and (9) into Eq. (8), we have

$$
\begin{aligned}
\Delta \gamma & \frac{\partial f}{\partial k_{0}}\left[2 x(1-x)+\frac{k_{1}}{\Delta \gamma}-\frac{k_{0}}{\Delta \gamma}\right]\left[\left(1-\frac{k_{1}}{\Delta \gamma}-\frac{k_{0}}{\Delta \gamma}\right)^{2}+\frac{4 k_{1}}{\Delta \gamma}\right] \\
= & \frac{2 x-1}{\Delta \gamma}\left(1+\frac{3 k_{1}}{\Delta \gamma}-\frac{k_{0}}{\Delta \gamma}\right)-x(1-x)\left[1+\frac{k_{1}}{\Delta \gamma}-\frac{3 k_{0}}{\Delta \gamma}+2\left(\frac{k_{1}+k_{0}}{\Delta \gamma}\right)^{2}\right] \\
& \quad-\frac{k_{1}}{\Delta \gamma}\left(1+\frac{k_{1}}{\Delta \gamma}-\frac{3 k_{0}}{\Delta \gamma}\right) .
\end{aligned}
$$

Note that one always has $\left[\left(1-k_{1} / \Delta \gamma-k_{0} / \Delta \gamma\right)^{2}+4 k_{1} / \Delta \gamma\right]>0$ and, in addition, $\left[2 x(1-x)+k_{1} / \Delta \gamma-k_{0} / \Delta \gamma\right]>0$ under the condition $\Delta \gamma>0$ and $k_{0}<k_{1}+\Delta \gamma / 2$. Consequently, we obtain the following relation

$$
\begin{aligned}
& \frac{2 x-1}{\Delta \gamma}\left(1+\frac{3 k_{1}}{\Delta \gamma}-\frac{k_{0, \mathrm{opt}}}{\Delta \gamma}\right)-x(1-x)\left[1+\frac{k_{1}}{\Delta \gamma}-\frac{3 k_{0, \mathrm{opt}}}{\Delta \gamma}+2\left(\frac{k_{1}+k_{0, \mathrm{opt}}}{\Delta \gamma}\right)^{2}\right] \\
& -\frac{k_{1}}{\Delta \gamma}\left(1+\frac{k_{1}}{\Delta \gamma}-\frac{3 k_{0, \mathrm{opt}}}{\Delta \gamma}\right)=0 .
\end{aligned}
$$

By employing Eq. (10), we will then generate the phase diagrams and discuss the implications in the next subsection.

Next, let us analytically derive the various asymptotes in the phase space.

(i) We will show that for $k_{1}=0, k_{0, \text { opt }} \rightarrow 1$ as $\Delta \gamma \rightarrow \infty$ and $k_{0, \mathrm{opt}} \rightarrow \Delta \gamma / 4$ in the small $\Delta \gamma$ limit. In the large $\Delta \gamma$ limit, we have $\rho^{-1}$ being a small number 
and thus $x_{1} \equiv x\left(k_{1}=0\right)=1-k_{0} / \Delta \gamma+\rho^{-1}\left[3-2 k_{0} / \Delta \gamma-\left(k_{0} / \Delta \gamma\right)^{-1}\right]+\cdots$. From $\left.\frac{\partial f}{\partial k_{0}}\right|_{k_{1}=0, k_{0}=k_{0, \text { opt }}}=0$, one gets

$$
\frac{2 x_{1}-1}{\Delta \gamma}-\left.x_{1}\left(1-x_{1}\right)\left(1-\frac{2 k_{0}}{\Delta \gamma}\right)\right|_{k_{0, \mathrm{opt}}}=0 .
$$

The 0 -th order (with respect to $\rho^{-1}$ ) solution is $\left(k_{0, \mathrm{opt}} / \Delta \gamma\right)^{2}-k_{0, \mathrm{opt}} / \Delta \gamma+1 / \Delta \gamma=0$. Thus, one has $k_{0, \text { opt }}=\Delta \gamma\left[1-(1-4 / \Delta \gamma)^{1 / 2}\right] / 2=1+(\Delta \gamma)^{-1}+\cdots$ in the large $\Delta \gamma$ limit and its value decreases to 1 as $\Delta \gamma \rightarrow \infty$. Subsequently, let us derive $k_{0 \text {,opt }}$ in the small $\Delta \gamma$ limit. Since $x_{1}(\Delta \gamma=0)=1 / 2$, we define $\epsilon \equiv x_{1}-1 / 2$ and expand $\epsilon$ as

$$
\begin{aligned}
\epsilon & =\frac{1}{2} \sum_{n=0}^{\infty}\left(\begin{array}{c}
1 / 2 \\
n+1
\end{array}\right)(-1)^{n} \frac{\left(1-\frac{2 k_{0}}{\Delta \gamma}\right)^{n+1}}{\left(1-\frac{k_{0}}{\Delta \gamma}\right)^{2 n+1}} \tanh ^{2 n+1}\left[\frac{\Delta \gamma}{2}\left(1-\frac{k_{0}}{\Delta \gamma}\right)\right] \\
& =\left(\frac{1-\frac{2 k_{0}}{\Delta \gamma}}{8}\right) \Delta \gamma-\frac{\left(1-\frac{2 k_{0}}{\Delta \gamma}\right)\left[1-\frac{2 k_{0}}{\Delta \gamma}+4\left(\frac{k_{0}}{\Delta \gamma}\right)^{2}\right]}{384}(\Delta \gamma)^{3}+O\left[(\Delta \gamma)^{5}\right] .
\end{aligned}
$$

On the other hand, from $\left.\frac{\partial f}{\partial k_{0}}\right|_{k_{1}=0, k_{0}=k_{0, \text { opt }}}=0$, one gets $\left(2 x_{1}-1\right) / \Delta \gamma=x_{1}\left(1-x_{1}\right)(1-$ $\left.2 k_{0} / \Delta \gamma\right)\left.\right|_{k_{0, \mathrm{opt}}}$ and thus $2 \epsilon=\left.\Delta \gamma\left(1 / 4-\epsilon^{2}\right)\left(1-2 k_{0} / \Delta \gamma\right)\right|_{k_{0, \mathrm{opt}}}$. Hence,

$$
\epsilon=\frac{-1 \pm\left[1+\frac{(\Delta \gamma)^{2}}{4}\left(1-\frac{2 k_{0, \mathrm{opt}}}{\Delta \gamma}\right)^{2}\right]^{1 / 2}}{\Delta \gamma\left(1-\frac{2 k_{0, \mathrm{opt}}}{\Delta \gamma}\right)} .
$$

From the fact $\epsilon(\Delta \gamma=0)=0$, the minus one is not acceptable. We then expand $\epsilon$ as

$$
\begin{aligned}
\epsilon & =\frac{\Delta \gamma}{4}\left(1-\frac{2 k_{0, \mathrm{opt}}}{\Delta \gamma}\right) \sum_{n=0}^{\infty}\left(\begin{array}{c}
1 / 2 \\
n+1
\end{array}\right)\left[\frac{(\Delta \gamma)^{2}}{4}\left(1-\frac{2 k_{0, \mathrm{opt}}}{\Delta \gamma}\right)^{2}\right]^{n} \\
& =\left(\frac{1-\frac{2 k_{0, \mathrm{opt}}}{\Delta \gamma}}{8}\right) \Delta \gamma-\frac{\left(1-\frac{2 k_{0, \mathrm{opt}}}{\Delta \gamma}\right)^{3}}{128}(\Delta \gamma)^{3}+O\left[(\Delta \gamma)^{5}\right] .
\end{aligned}
$$

With Eq. (11) = Eq. (12), one gets

$$
\frac{\left(1-\frac{2 k_{0, \mathrm{opt}}}{\Delta \gamma}\right)^{3}}{128}=\frac{\left(1-\frac{2 k_{0, \mathrm{opt}}}{\Delta \gamma}\right)\left[1-\frac{2 k_{0, \mathrm{opt}}}{\Delta \gamma}+4\left(\frac{k_{0, \mathrm{opt}}}{\Delta \gamma}\right)^{2}\right]}{384}+O\left[(\Delta \gamma)^{2}\right] .
$$

Thus, for $k_{1}=0, k_{0, \text { opt }}=\Delta \gamma / 4+O\left[(\Delta \gamma)^{3}\right]$ in the small $\Delta \gamma$ limit.

(ii) We will show that $k_{0, \text { opt }} \rightarrow 1-k_{1}$ for $0 \leq k_{1} \leq 1$ as $\Delta \gamma \rightarrow \infty$. From Eq. (7), one has

$$
2 \Delta \gamma \frac{\partial f}{\partial k_{0}}+1=\frac{\partial}{\partial k_{0}}\left\{\ln \left[\frac{\left(\tilde{f}_{+}-1+x\right)\left(1-x-\tilde{f}_{-}\right)}{\left(\tilde{f}_{+}-x\right)\left(x-\tilde{f}_{-}\right)}\right]\right\} .
$$

By using $\rho^{-1}$ as the small parameter for expansion, $x$ can be recast as

$$
x=\tilde{f}_{+}+\frac{\left(1-2 \tilde{f}_{+}\right)\left(\tilde{f}_{+}-\tilde{f}_{-}\right)}{1-\tilde{f}_{+}-\tilde{f}_{-}} \rho^{-1}+O\left(\rho^{-2}\right) .
$$


By substituting the above expression for $x$ into Eq. (13), one gets

$$
0=\left.\frac{\partial f}{\partial k_{0}}\right|_{k_{0}=k_{0, \mathrm{opt}}} \approx \frac{\partial \tilde{f}_{+}}{\partial k_{0}}+\left.\frac{1}{\Delta \gamma} \frac{\partial}{\partial k_{0}}\left[\ln \left(\frac{1-\tilde{f}_{+}-\tilde{f}_{-}}{\tilde{f}_{+}-\tilde{f}_{-}}\right)\right]\right|_{k_{0}=k_{0, \mathrm{opt}}} .
$$

With some calculation, we derive

$$
\begin{aligned}
0 \approx & k_{1}\left(k_{1}+k_{0, \mathrm{opt}}\right)^{2}\left[\left(\Delta \gamma-k_{1}-k_{0, \mathrm{opt}}\right)^{2}+4 k_{1} \Delta \gamma\right]+\Delta \gamma\left(\Delta \gamma+3 k_{1}-k_{0, \mathrm{opt}}\right)^{2} \\
& -\left(k_{1}+k_{0, \mathrm{opt}}\right)\left(\Delta \gamma+3 k_{1}-k_{0, \mathrm{opt}}\right)\left[\left(\Delta \gamma-k_{1}-k_{0, \mathrm{opt}}\right)^{2}+4 k_{1} \Delta \gamma\right] .
\end{aligned}
$$

The sum of the terms in the leading order is given by $\left(1-k_{1}-k_{0, \text { opt }}\right)(\Delta \gamma)^{3}+O\left[(\Delta \gamma)^{2}\right]=$ 0 . Thus, $k_{0, \text { opt }} \rightarrow 1-k_{1}$ for $0 \leq k_{1} \leq 1$ as $\Delta \gamma \rightarrow \infty$.

(iii) We will derive the phase boundary of $k_{0, \text { opt }}=0$ in the large $\Delta \gamma$ limit and the small $\Delta \gamma$ limit. In the large $\Delta \gamma$ limit, we can use $\rho^{-1}$ as the small parameter for expansion. From Eq. (6),

$$
x_{0} \equiv x\left(k_{0}=0\right)=1-\left(\frac{\Delta \gamma}{k_{1}}+1\right) \rho^{-1}+\cdots .
$$

From $\left.\frac{\partial f}{\partial k_{0}}\right|_{k_{0}=0}=0$, one gets

$$
0=\frac{2 x_{0}-1}{\Delta \gamma}\left(1+3 \frac{k_{1}}{\Delta \gamma}\right)-x_{0}\left(1-x_{0}\right)\left[1+\frac{k_{1}}{\Delta \gamma}+2\left(\frac{k_{1}}{\Delta \gamma}\right)^{2}\right]-\frac{k_{1}}{\Delta \gamma}\left(1+\frac{k_{1}}{\Delta \gamma}\right) .
$$

The 0 -th order (with respect to $\left.\rho^{-1}\right)$ solution is $\left(k_{1} / \Delta \gamma\right)^{2}+(1-3 / \Delta \gamma) k_{1} / \Delta \gamma-1 / \Delta \gamma=$ 0 . Hence, $k_{1}=1+O\left[(\Delta \gamma)^{-1}\right]+\cdots$. Consequently, the phase boundary for $k_{0, \mathrm{opt}}=0$ in the large $\Delta \gamma$ limit is as follows: $k_{1}$ decreases to the asymptote $k_{1}=1$ as $\Delta \gamma \rightarrow \infty$. Subsequently, let us derive the phase boundary in the small $\Delta \gamma$ limit. Since $x_{0}(\Delta \gamma=$ $0)=1 / 2$, we define $\epsilon_{0} \equiv x_{0}-1 / 2$ and expand $\epsilon_{0}$ as

$$
\begin{aligned}
\epsilon_{0}= & \frac{1}{2} \sum_{n=0}^{\infty}\left(\begin{array}{c}
1 / 2 \\
n+1
\end{array}\right)(-1)^{n} \frac{\left(1+\frac{2 k_{1}}{\Delta \gamma}\right)^{n+1}}{\left(1+\frac{k_{1}}{\Delta \gamma}\right)^{2 n+1}} \tanh ^{2 n+1}\left[\frac{\Delta \gamma}{2}\left(1+\frac{k_{1}}{\Delta \gamma}\right)\right] \\
= & \frac{1+\frac{2 k_{1}}{\Delta \gamma}}{8} \Delta \gamma-\frac{\left(1+\frac{2 k_{1}}{\Delta \gamma}\right)\left[1+\frac{2 k_{1}}{\Delta \gamma}+4\left(\frac{k_{1}}{\Delta \gamma}\right)^{2}\right]}{384}(\Delta \gamma)^{3} \\
& +\frac{\left(1+\frac{2 k_{1}}{\Delta \gamma}\right)\left[1+\frac{4 k_{1}}{\Delta \gamma}+6\left(\frac{k_{1}}{\Delta \gamma}\right)^{2}+4\left(\frac{k_{1}}{\Delta \gamma}\right)^{3}+16\left(\frac{k_{1}}{\Delta \gamma}\right)^{4}\right]}{15360}(\Delta \gamma)^{5}+\cdots
\end{aligned}
$$

On the other hand, from $\left.\frac{\partial f}{\partial k_{0}}\right|_{k_{0}=0}=0$, one gets $\left(2 x_{0}-1\right)\left(1+3 k_{1} / \Delta \gamma\right) / \Delta \gamma-x_{0}(1-$ $\left.x_{0}\right)\left[1+k_{1} / \Delta \gamma+2\left(k_{1} / \Delta \gamma\right)^{2}\right]-\left(k_{1} / \Delta \gamma\right)\left(1+k_{1} / \Delta \gamma\right)=0$ and thus

$$
\epsilon_{0}=\frac{-\left(1+\frac{3 k_{1}}{\Delta \gamma}\right) \pm\left\{\left(1+\frac{3 k_{1}}{\Delta \gamma}\right)^{2}+\frac{(\Delta \gamma)^{2}}{4}\left[1+\frac{k_{1}}{\Delta \gamma}+2\left(\frac{k_{1}}{\Delta \gamma}\right)^{2}\right]\left(1+\frac{2 k_{1}}{\Delta \gamma}\right)\left(1+\frac{3 k_{1}}{\Delta \gamma}\right)\right\}^{1 / 2}}{\Delta \gamma\left[1+\frac{k_{1}}{\Delta \gamma}+2\left(\frac{k_{1}}{\Delta \gamma}\right)^{2}\right]} .
$$


From the fact $\epsilon_{0}(\Delta \gamma=0)=0$, the minus one is not acceptable. We then expand $\epsilon_{0}$ as

$$
\begin{aligned}
\epsilon_{0}= & \frac{\Delta \gamma\left(1+\frac{2 k_{1}}{\Delta \gamma}\right)}{4} \sum_{n=0}^{\infty}\left(\begin{array}{c}
1 / 2 \\
n+1
\end{array}\right)\left\{\frac{(\Delta \gamma)^{2}\left[1+\frac{k_{1}}{\Delta \gamma}+2\left(\frac{k_{1}}{\Delta \gamma}\right)^{2}\right]\left(1+\frac{2 k_{1}}{\Delta \gamma}\right)}{4\left(1+\frac{3 k_{1}}{\Delta \gamma}\right)}\right\}^{n} \\
= & \left(\frac{1+\frac{2 k_{1}}{\Delta \gamma}}{8}\right) \Delta \gamma-\frac{\left[1+\frac{k_{1}}{\Delta \gamma}+2\left(\frac{k_{1}}{\Delta \gamma}\right)^{2}\right]\left(1+\frac{2 k_{1}}{\Delta \gamma}\right)^{2}}{128\left(1+\frac{3 k_{1}}{\Delta \gamma}\right)}(\Delta \gamma)^{3} \\
& +\frac{\left[1+\frac{k_{1}}{\Delta \gamma}+2\left(\frac{k_{1}}{\Delta \gamma}\right)^{2}\right]^{2}\left(1+\frac{2 k_{1}}{\Delta \gamma}\right)^{3}}{1024\left(1+\frac{3 k_{1}}{\Delta \gamma}\right)^{2}}(\Delta \gamma)^{5}+\cdots
\end{aligned}
$$

From Eq. (14) = Eq. (15), one has

$$
\begin{gathered}
-\frac{\left[1+\frac{2 k_{1}}{\Delta \gamma}+4\left(\frac{k_{1}}{\Delta \gamma}\right)^{2}\right]}{384}+\frac{\left[1+\frac{4 k_{1}}{\Delta \gamma}+6\left(\frac{k_{1}}{\Delta \gamma}\right)^{2}+4\left(\frac{k_{1}}{\Delta \gamma}\right)^{3}+16\left(\frac{k_{1}}{\Delta \gamma}\right)^{4}\right]}{15360}(\Delta \gamma)^{2}+\cdots \\
=-\frac{\left[1+\frac{k_{1}}{\Delta \gamma}+2\left(\frac{k_{1}}{\Delta \gamma}\right)^{2}\right]\left(1+\frac{2 k_{1}}{\Delta \gamma}\right)}{128\left(1+\frac{3 k_{1}}{\Delta \gamma}\right)}+\frac{\left[1+\frac{k_{1}}{\Delta \gamma}+2\left(\frac{k_{1}}{\Delta \gamma}\right)^{2}\right]\left(1+\frac{2 k_{1}}{\Delta \gamma}\right)^{2}}{1024\left(1+\frac{3 k_{1}}{\Delta \gamma}\right)^{2}}(\Delta \gamma)^{2}+\cdots
\end{gathered}
$$

With some calculation, we obtain the phase boundary of $k_{0, \text { opt }}=0$ in the small $\Delta \gamma$ limit: $k_{1}=(5 \Delta \gamma / 2)^{1 / 3}+O(\Delta \gamma)$.

\subsubsection{Numerical results}

For verification, we numerically generate the phase diagram of $k_{0, \text { opt }}$ versus $\Delta \gamma$ and $k_{1}$, which are given in Figs. 2 and 3, respectively. Figure 2 demonstrates that the value of $k_{0 \text {,opt }}$ as a function of $\Delta \gamma$ (with $k_{1}$ fixed) can be characterized into four different regimes: $k_{1}=0,0<k_{1} \leq 1,1<k_{1}<1.40$, and $k_{1} \geq 1.40$. With $k_{1}=0$, the value of $k_{0, \text { opt }} \approx \Delta \gamma / 4$ for $\Delta \gamma \ll 1$, then increases until reaching a maximum, and then decreases to 1 as $\Delta \gamma \rightarrow$ $\infty$. With $0<k_{1} \leq 1$, the value of $k_{0 \text {,opt }}=0$ for $\Delta \gamma \leq 2 k_{1}^{3} / 5+O\left(k_{1}^{5}\right)$, then increases until reaching a maximum, and then decreases to $1-k_{1}$ as $\Delta \gamma \rightarrow \infty$. With $1<k_{1}<1.40$, $k_{0, \text { opt }}=0$ for small $\Delta \gamma$, then increases until reaching a maximum, and then decreases to 0 as $\Delta \gamma \rightarrow \infty$. With $k_{1} \geq 1.40, k_{0 \text {,opt }}=0$ for arbitrary $\Delta \gamma$. In contrast, the value of $k_{0 \text {,opt }}$ is always a strictly decreasing function of $k_{1}$ for any fixed value of $\Delta \gamma$ as shown in Fig. 3. All the analytically derived asymptotes in the phase space are numerically verified. For example, the analytically obtained phase boundary of $k_{0, \text { opt }}=0$ in the small $\Delta \gamma$ limit, $k_{1}=(5 \Delta \gamma / 2)^{1 / 3}+O(\Delta \gamma)$, is fully numerically confirmed and illustrated in the inset of Fig. 2. Notice that the dashed line $\left(k_{1}=0.7\right)$ in the inset of Fig. 2 kicks out right at $\Delta \gamma \approx 2 k_{1}^{3} / 5$. In addition, the inset of Fig. 2 also confirms our analytical derivation: $k_{0, \text { opt }} \rightarrow \Delta \gamma / 4$ for $k_{1}=0$ in the small $\Delta \gamma$ limit. Besides, the analytically derived property, $k_{0, \text { opt }} \rightarrow 1-k_{1}$ for $0 \leq k_{1} \leq 1$ as $\Delta \gamma \rightarrow \infty$, is also numerically confirmed and shown in Fig. 3. Note that Fig. 3 demonstrates that, in the large $\Delta \gamma$ limit, the convergence of $k_{0 \text {,opt }}$ toward $1-k_{1}$ is very fast.

The most intriguing feature of this system is that $k_{0, \text { opt }}>0$ in some ranges of $\Delta \gamma$ and $k_{1}$. It can be understood as follows. Recall that the environment switches between two states periodically and the favorable cell population will become unfavorable after 


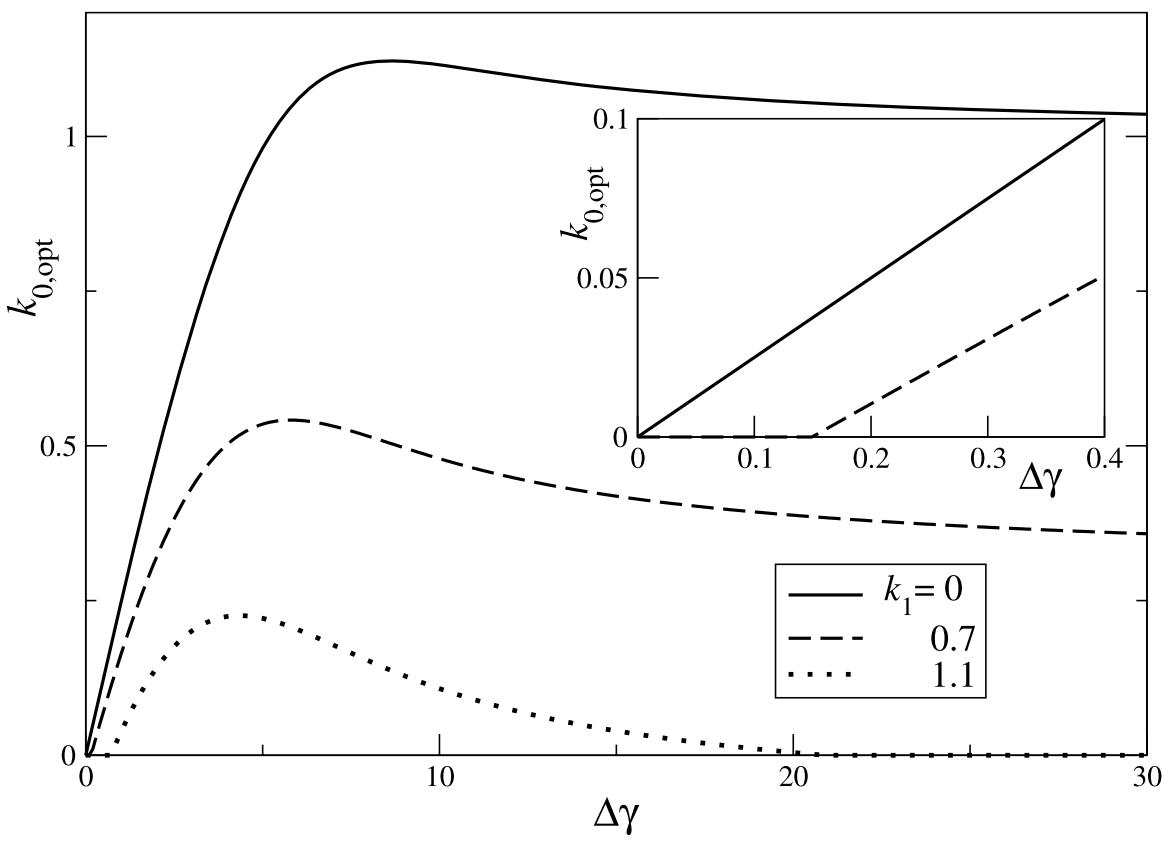

Fig. 2 Growth of cell populations in periodically switching environments with symmetrical time-span. We plot $k_{0, \text { opt }}$ vs $\Delta \gamma$ with a fixed $k_{1}$ in three different regimes: $k_{1}=0$ (solid curve), $0<k_{1}(=0.7) \leq 1$ (dashed curve), and $1<k_{1}(=1.1)<1.4$ (dotted curve). See the inset for the small $\Delta \gamma$ behavior of $k_{0 \text {,opt }}$ with $k_{1}=0$ (solid curve) and $0<k_{1}(=0.7) \leq 1$ (dashed curve). For $k_{1} \geq 1.4, k_{0 \text {,opt }}$ is always zero.

the flipping of environmental states. Hence, a high value of $\tilde{f}_{+}$, the steady state solution of $f_{1}(t)$, plays the role of a double-edged knife. That is, a high value of $\tilde{f}_{+}$corresponds to a high value of the favorable cell population at the moment right before the flipping of the environment state and also a high value of the unfavorable cell population at the moment right after the flipping of the environment state. Thus, one needs a balance between $\Delta \gamma$ (the difference in the growth rate per capita between the fit and unfit cell states) and $k_{0}$ for the system to reach a higher value of $f$ (the overall population fitness), if $k_{1}$ is fixed. This explains the unimodal shape of $k_{0, \text { opt }}$ versus $\Delta \gamma$, for a fixed $k_{1}$ value smaller than a threshold value 1.40 . This threshold value is numerically obtained. On the other hand, as the value of $k_{1}$ increases, the switching rate from the unfit cell state to the fit cell state increases, which corresponds to a smaller response time for the system. This explains why $k_{0, \text { opt }}$ is a strictly decreasing function of $k_{1}$ for any fixed value of $\Delta \gamma$. For the value of $k_{1}$ smaller than the threshold value 1.40 , the interplay of $k_{1}, k_{0}$, and $\Delta \gamma$ makes the phase diagrams quite complicated. To be more specific, we would like to give a mathematical reasoning as follows. Define $T_{1}$ and $T_{2}$ to be the time durations for $f_{1, \text { per }}(t)$ from $f_{1, \text { per }}\left(t=n^{+}\right)$rising to $\left(\tilde{f}_{+}+\tilde{f}_{-}\right) / 2$ and from $\left(\tilde{f}_{+}+\tilde{f}_{-}\right) / 2$ rising to $f_{1, \mathrm{per}}[t=(n+$ $\left.1)^{-}\right]$, respectively. (See Fig. 1 for illustration.) We have $T_{1}=\ln \left[\left(\tilde{f}_{+}-1+x\right) /(1-x-\right.$ $\left.\left.\tilde{f}_{-}\right)\right] /\left[\Delta \gamma\left(\tilde{f}_{+}-\tilde{f}_{-}\right)\right]$and $T_{2}=\ln \left[\left(x-\tilde{f}_{-}\right) /\left(\tilde{f}_{+}-x\right)\right] /\left[\Delta \gamma\left(\tilde{f}_{+}-\tilde{f}_{-}\right)\right]$. (Although, for $f_{1, \text { per }}\left(t=n^{+}\right)>\left(\tilde{f}_{+}+\tilde{f}_{-}\right) / 2$, we will have $T_{1}<0$ and $T_{2}>1$, our following argument is still applicable. Just take it as a fictitious extension to the negative time.) During the first 


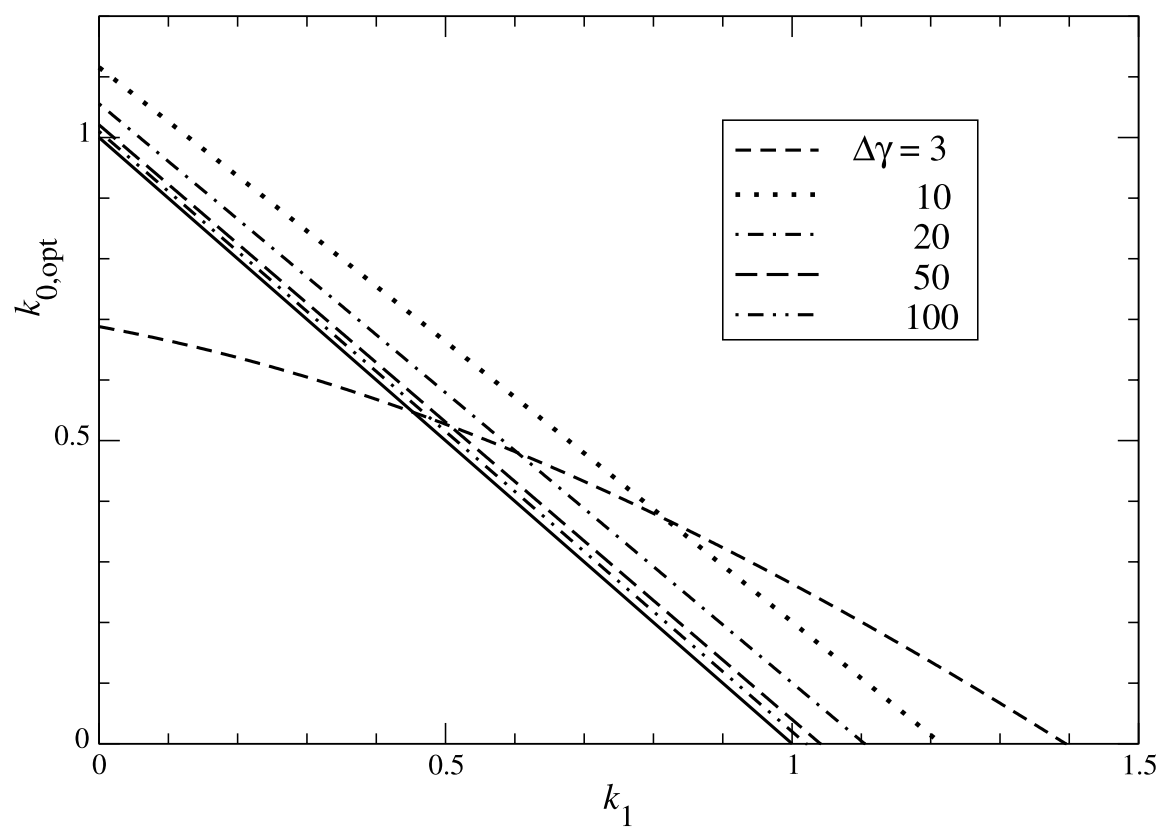

Fig. 3 A plot of $k_{0 \text {,opt }}$ vs $k_{1}$ with $\Delta \gamma=3,10,20,50$, and 100, for the cell populations in periodically switching environments with symmetrical time-span. The solid line represents the analytically obtained asymptote: $k_{0, \text { opt }} \rightarrow 1-k_{1}$ for $0 \leq k_{1} \leq 1$ as $\Delta \gamma \rightarrow \infty$.

time interval $T_{1}, f_{1, \text { per }}(t)$ spends longer time near $f_{1, \text { per }}\left(t=n^{+}\right)=1-x$ and less time near $\left(\tilde{f}_{+}+\tilde{f}_{-}\right) / 2$ as can be seen easily from the rate $d f_{1} / d t$. Similarly, during the second time interval $T_{2}, f_{1, \mathrm{per}}(t)$ spends longer time near $f_{1, \mathrm{per}}\left[t=(n+1)^{-}\right]=x$ and less time near $\left(\tilde{f}_{+}+\tilde{f}_{-}\right) / 2$. We have $\left(\tilde{f}_{+}+\tilde{f}_{-}\right) / 2<1 / 2$, so that we always have $T_{2}>T_{1} \cdot f_{1, \text { per }}(t)$ always spends longer time around $x$ than around $1-x$. For this effect alone, the overall fitness $f$ is an increasing function of $x$, which is a decreasing function of $k_{0}$. On the other hand, to achieve a larger overall fitness $f$, we expect a larger difference between $T_{2}$ and $T_{1}$ (i.e., $\left.T_{2}-T_{1}=2 \ln \left[\left(x-\tilde{f}_{-}\right) /\left(\tilde{f}_{+}-x\right)\right] /\left[\Delta \gamma\left(\tilde{f}_{+}-\tilde{f}_{-}\right)\right]-1\right)$, which is an increasing function of $k_{0}$. A compromise will be reached between the above two effects to achieve an optimal value of $k_{0}$. For illustration, we consider the case with sufficiently large $\Delta \gamma$ so that $1-x<\left(\tilde{f}_{+}+\tilde{f}_{-}\right) / 2$. We further make the following approximation: during the first time interval $T_{1}, f_{1, \text { per }}(t)$ takes the value $1-x$ and, during the second time interval $T_{2}, f_{1, \text { per }}(t)$ takes the value $x$. After some calculation, we have

$$
f \approx \frac{1}{2}+\left(\frac{1}{2}-\frac{k_{0}}{\Delta \gamma}\right)\left[1+\frac{2}{\Delta \gamma+k_{1}-k_{0}} \ln \left(\frac{k_{0}+k_{1}}{\Delta \gamma}\right)\right] .
$$

Obviously, the factor $1 / 2-k_{0} / \Delta \gamma$ is a decreasing function of $k_{0}$, while the other factor $\left\{1+2 \ln \left[\left(k_{0}+k_{1}\right) /(\Delta \gamma)\right] /\left(\Delta \gamma+k_{1}-k_{0}\right)\right\}$ is an increasing function of $k_{0}$. From $\partial f / \partial k_{0}=0$, we have $k_{0, \text { opt }}=1-k_{1}$ under the conditions $0 \leq k_{1} \leq 1$ and $\Delta \gamma \rightarrow \infty$. 


\section{Time-dependent cellular transitions}

In the minimal dynamical model proposed by Thattai and van Oudenaarden (2004), cellular transitions are assumed to be instantaneous. However, cellular transitions in reality do cost time. In this section, we will consider the switching dynamics of cell populations with the cellular transition time explicitly taken into account. Assume that a duration $t_{1}$ $\left(t_{0}\right)$ is needed for a cell to make the transition from state $c_{0}$ to state $c_{1}$ (state $c_{1}$ to state $c_{0}$ ). During this time interval, the proteins pertaining to the new state are being synthesized and those not pertaining to this new state are being degraded. In this transition period, the protein content of the cell has not settled down to one of its stable states. Thus, the growth rate per capita during this period is expected to take a much lower value $\zeta_{1}\left(\zeta_{0}\right)$ instead of $\gamma_{1}\left(\gamma_{0}\right)$. Introduce the notation $\tilde{n}_{1}\left(\tilde{n}_{0}\right)$ representing the number of cells whose genes for the state $c_{1}\left(c_{0}\right)$ have been turned on but protein contents have not settled down to the stable state $c_{1}\left(c_{0}\right)$. The dynamical equations should be modified as follows:

$$
\begin{aligned}
& \frac{d n_{1}}{d t}=\gamma_{1}\left(n_{1}-\tilde{n}_{1}\right)+\zeta_{1} \tilde{n}_{1}-k_{0}\left(n_{1}-\tilde{n}_{1}\right)+k_{1}\left(n_{0}-\tilde{n}_{0}\right), \\
& \frac{d n_{0}}{d t}=\gamma_{0}\left(n_{0}-\tilde{n}_{0}\right)+\zeta_{0} \tilde{n}_{0}-k_{1}\left(n_{0}-\tilde{n}_{0}\right)+k_{0}\left(n_{1}-\tilde{n}_{1}\right) .
\end{aligned}
$$

With the assumption $\gamma_{1} t_{1}, \gamma_{0} t_{0} \ll 1$, we have $\tilde{n}_{1} \approx\left(n_{0}-\tilde{n}_{0}\right) k_{1} t_{1}$ and $\tilde{n}_{0} \approx\left(n_{1}-\tilde{n}_{1}\right) k_{0} t_{0}$. Hence, $\tilde{n}_{1} \approx \frac{k_{1} t_{1}\left(n_{0}-k_{0} t_{0} n_{1}\right)}{1-k_{1} t_{1} k_{0} t_{0}}$ and $\tilde{n}_{0} \approx \frac{k_{0} t_{0}\left(n_{1}-k_{1} t_{1} n_{0}\right)}{1-k_{1} t_{1} t_{0} t_{0}}$. By substituting the expressions of $\tilde{n}_{1}$ and $\tilde{n}_{0}$ into the above modified dynamical equations and performing some calculation, the modified dynamical equations can be recast in the following form:

$$
\begin{aligned}
& \frac{d n_{1}}{d t}=\gamma_{1}^{\prime} n_{1}-k_{0}^{\prime} n_{1}+k_{1}^{\prime} n_{0}, \\
& \frac{d n_{0}}{d t}=\gamma_{0}^{\prime} n_{0}-k_{1}^{\prime} n_{0}+k_{0}^{\prime} n_{1},
\end{aligned}
$$

with the effective parameters

$$
\begin{aligned}
k_{1}^{\prime} & =\frac{k_{1}+\left(\zeta_{1}-\gamma_{1}+k_{0}\right) k_{1} t_{1}}{1-k_{1} t_{1} k_{0} t_{0}}, \\
k_{0}^{\prime} & =\frac{k_{0}+\left(\zeta_{0}-\gamma_{0}+k_{1}\right) k_{0} t_{0}}{1-k_{1} t_{1} k_{0} t_{0}}, \\
\gamma_{1}^{\prime} & =\gamma_{1}+\frac{\left(\zeta_{0}-\gamma_{0}\right) k_{0} t_{0}-\left(\zeta_{1}-\gamma_{1}\right) k_{1} t_{1} k_{0} t_{0}}{1-k_{1} t_{1} k_{0} t_{0}}, \\
\gamma_{0}^{\prime} & =\gamma_{0}+\frac{\left(\zeta_{1}-\gamma_{1}\right) k_{1} t_{1}-\left(\zeta_{0}-\gamma_{0}\right) k_{1} t_{1} k_{0} t_{0}}{1-k_{1} t_{1} k_{0} t_{0}} .
\end{aligned}
$$

If the values of $k_{1} t_{1}$ and $k_{0} t_{0}$ are much less than 1 , the above effective parameters can be approximated as (to the leading order of $k_{1} t_{1}$ and $k_{0} t_{0}$ )

$$
\begin{aligned}
& k_{1}^{\prime} \approx k_{1}+\left(\zeta_{1}-\gamma_{1}+k_{0}\right) k_{1} t_{1}, \\
& k_{0}^{\prime} \approx k_{0}+\left(\zeta_{0}-\gamma_{0}+k_{1}\right) k_{0} t_{0},
\end{aligned}
$$




$$
\begin{aligned}
& \gamma_{1}^{\prime} \approx \gamma_{1}+\left(\zeta_{0}-\gamma_{0}\right) k_{0} t_{0}, \\
& \gamma_{0}^{\prime} \approx \gamma_{0}+\left(\zeta_{1}-\gamma_{1}\right) k_{1} t_{1} .
\end{aligned}
$$

Note that the mathematical forms of the above modified dynamical equations are exactly the same as those of Eqs. (1) and (2). The only assumption we have made in the above derivation is $\gamma_{1} t_{1}\left(\gamma_{0} t_{0}\right) \ll 1$. Actually, for any cell populations satisfying $\Delta \gamma t_{1}\left(\Delta \gamma t_{0}\right) \ll$ 1 , the above result is generally applicable, by introducing $\bar{\gamma} \equiv\left(\gamma_{1}+\gamma_{0}\right) / 2$ and replacing the parameters $\left(\gamma_{1}, \gamma_{0}, \zeta_{1}, \zeta_{0}\right)$ with $\left(\gamma_{1}-\bar{\gamma}, \gamma_{0}-\bar{\gamma}, \zeta_{1}-\bar{\gamma}, \zeta_{0}-\bar{\gamma}\right)$. Note that this operation just makes the time-dependent cell populations $\left(n_{1}\right.$ and $\left.n_{0}\right)$ reduced by a factor $e^{\bar{\gamma} t}$, but the fraction of cells in the fit (unfit) state will not be changed.

Subsequently, let us consider some special biological situations, in which a significant part of cell populations can not survive the cellular transition period. Thus, the growth rates per capita during this period, $\zeta_{1}$ and $\zeta_{0}$, are expected to take negative values with $\left|\zeta_{1} t_{1}\right|$ and $\left|\zeta_{0} t_{0}\right|$ not much less than 1 . Let $\tilde{n}_{1}\left(\tilde{n}_{0}\right)$ denote the number of cells which make transition to the new cell state during the time interval $\left[t-t_{1}, t\right]\left(\left[t-t_{0}, t\right]\right)$ and are alive through this period of time. With some calculation, we have

$$
\begin{aligned}
\tilde{n}_{1}(t) & =k_{1} \int_{0}^{t_{1}}\left[n_{0}(t-\tau)-\tilde{n}_{0}(t-\tau)\right] e^{\zeta_{1} \tau} d \tau \\
& \approx\left[n_{0}(t)-\tilde{n}_{0}(t)\right] k_{1} \int_{0}^{t_{1}} e^{\zeta_{1} \tau} d \tau \\
& =\left[n_{0}(t)-\tilde{n}_{0}(t)\right] k_{1} t_{1} \xi_{1}
\end{aligned}
$$

and

$$
\begin{aligned}
\tilde{n}_{0}(t) & =k_{0} \int_{0}^{t_{0}}\left[n_{1}(t-\tau)-\tilde{n}_{1}(t-\tau)\right] e^{\zeta_{0} \tau} d \tau \\
& \approx\left[n_{1}(t)-\tilde{n}_{1}(t)\right] k_{0} \int_{0}^{t_{0}} e^{\zeta_{0} \tau} d \tau \\
& =\left[n_{1}(t)-\tilde{n}_{1}(t)\right] k_{0} t_{0} \xi_{0}
\end{aligned}
$$

with $\xi_{1} \equiv\left(1-e^{-\left|\zeta_{1} t_{1}\right|}\right) /\left|\zeta_{1} t_{1}\right|$ and $\xi_{0} \equiv\left(1-e^{-\left|\zeta_{0} t_{0}\right|}\right) /\left|\zeta_{0} t_{0}\right|$. The corresponding dynamical equations will still take the same mathematical forms as those of Eqs. (1) and (2), but the effective parameters will be modified as

$$
\begin{aligned}
k_{1}^{\prime} & =\frac{k_{1}+\left(\zeta_{1}-\gamma_{1}+k_{0}\right) \xi_{1} k_{1} t_{1}}{1-\xi_{1} \xi_{0} k_{1} t_{1} k_{0} t_{0}}, \\
k_{0}^{\prime} & =\frac{k_{0}+\left(\zeta_{0}-\gamma_{0}+k_{1}\right) \xi_{0} k_{0} t_{0}}{1-\xi_{1} \xi_{0} k_{1} t_{1} k_{0} t_{0}}, \\
\gamma_{1}^{\prime} & =\gamma_{1}+\frac{\left(\zeta_{0}-\gamma_{0}\right) \xi_{0} k_{0} t_{0}-\left(\zeta_{1}-\gamma_{1}\right) \xi_{1} \xi_{0} k_{1} t_{1} k_{0} t_{0}}{1-\xi_{1} \xi_{0} k_{1} t_{1} k_{0} t_{0}}, \\
\gamma_{0}^{\prime} & =\gamma_{0}+\frac{\left(\zeta_{1}-\gamma_{1}\right) \xi_{1} k_{1} t_{1}-\left(\zeta_{0}-\gamma_{0}\right) \xi_{1} \xi_{0} k_{1} t_{1} k_{0} t_{0}}{1-\xi_{1} \xi_{0} k_{1} t_{1} k_{0} t_{0}} .
\end{aligned}
$$


If the values of $\xi_{1} k_{1} t_{1}$ and $\xi_{0} k_{0} t_{0}$ are much less than 1 , these effective parameters can be approximated as (to the leading order of $\xi_{1} k_{1} t_{1}$ and $\xi_{0} k_{0} t_{0}$ )

$$
\begin{aligned}
& k_{1}^{\prime} \approx k_{1}+\left(\zeta_{1}-\gamma_{1}+k_{0}\right) \xi_{1} k_{1} t_{1}, \\
& k_{0}^{\prime} \approx k_{0}+\left(\zeta_{0}-\gamma_{0}+k_{1}\right) \xi_{0} k_{0} t_{0}, \\
& \gamma_{1}^{\prime} \approx \gamma_{1}+\left(\zeta_{0}-\gamma_{0}\right) \xi_{0} k_{0} t_{0}, \\
& \gamma_{0}^{\prime} \approx \gamma_{0}+\left(\zeta_{1}-\gamma_{1}\right) \xi_{1} k_{1} t_{1} .
\end{aligned}
$$

In words, we have considered various biological situations with the cellular transition time explicitly taken into account. Interestingly, we find that the systems with time-dependent cellular transitions collapse to the earlier set of dynamical equations, Eqs. (1) and (2), with the modified parameters. So, our forgoing analysis in Section 2 is generally applicable here.

\section{The analogy to stochastic resonance}

Interestingly, the problem we discuss in this paper bears much resemblance to a renowned phenomenon, stochastic resonance, in physics and engineering (Gammaitoni et al., 1998; Moss, 1994). In recent years, scientists have found great applications of stochastic resonance ranging from laser technology, semiconductors, electronic devices, superconducting quantum interference devices, even to the noise-assisted information flow in the sensory neurons of various animals. However, except the neuronal systems, stochastic resonance has not yet been discussed in biological systems. Here, we would like to point out that the long-term optimal fitness of cells in a periodically switching environment can be viewed as self-optimization exploiting stochastic resonance.

Let us first explain the mechanism of stochastic resonance by the following example as an illustration. Consider the one-dimensional motion of a heavily damped particle under a double-well potential along the $x$-axis. The potential minima are located at $\pm x_{m}$, separated by a potential barrier. A weak external periodic force is applied to the particle such that the double-well potential is tilted up and down. The weak periodic force plays the role of a small perturbation to the original system, which is too weak to make the particle roll periodically from one well to the other. Now let the whole system be bathed in a thermal reservoir and thus subject to the random forces due to thermal fluctuations (consistent with the microscopic view of modern physical kinetic theory and chemical reaction-rate theory). These random forces due to thermal fluctuations enable the particle to hop over the potential barrier and reach the global energy minimum with some probability. In the steady state, the mean value (i.e., the ensemble average) of the position of the particle, $\langle x(t)\rangle$, is a periodic function of time. The amplitude of $\langle x(t)\rangle$ is denoted by $x_{0}$. The magnitude of $x_{0}$ depends on the relations among the noise strength, the period and the strength of the external periodic force, and the height of the potential barrier. One looks for the conditions for $x_{0}$ to reach the maximum. Roughly speaking, $x_{0}$ will reach the maximum if the mean residence time of the particle in each well (or the mean first passage time) matches the half-period of the external periodic force. This is the celebrated stochastic resonance effect. Namely, the periodic response of the system can be manipulated by changing the noise strength. If one is only concerned about the hopping between 
the two potential wells and the intrawell motion is negligible, a two-state model is a good reduction of the original continuous dynamics of the bistable process. Physically, this corresponds to the situation in which the period of eternal periodic force is much larger than the time scale for the intrawell relaxation of the particle. Hence, one obtains the following master equation

$$
\frac{d n_{ \pm}}{d t}=-W_{\mp} n_{ \pm}+W_{ \pm} n_{\mp}
$$

in which $n_{ \pm}$denotes the probability for the particle being in the potential well with the potential minimum located at $\pm x_{m} . W_{\mp}$ denotes the corresponding transition rate.

Next, we will explain in detail how the problem about the long-term fitness of cells in a periodically switching environment bears resemblance to the physical processes of stochastic resonance. Since the environment switches between the two environmental states $e_{a}$ and $e_{b}$, cells thus make switches between the cell states $c_{a}$ and $c_{b}$ to fit their living environments. The cell-state switching for an individual cell is actually a switch between different genetic expressions. The general mechanism of a genetic switch in the microscopic molecular level is given as follows. The switch DNA element contains several operator sites, a single promoter site, and a few subunit genes. The RNA polymerase bound to the promoter site directs genetic transcription and the transcription rate is controlled by the regulatory proteins. The regulatory proteins bind directly to the operator sites of the switch DNA. These regulatory proteins can either activate or repress gene transcription and translation. Thus, a genetic switch involves a sequence of coupled biochemical reactions. Note that all the biochemical reactions are intrinsically stochastic. The stochasticity of biochemical kinetics is a consequence of the fact that collisions in a system of molecules in thermal equilibrium occur in an essentially random manner. The reactant molecules must first collide and form an activated complex. Then, the activated complex can decay into the products. The amount of energy which must be absorbed to form the activated complex is called the activation energy. Thus, the activation energy plays the role of an effective potential barrier. For the occurrence of a biochemical reaction, the reactant system must couple to a thermal reservoir and then gain the needed thermal fluctuational energy to overcome the effective potential barrier. Hence, in terms of the picture of the effective potential energy, the genetic switch of an individual cell can be viewed as a cell making transitions between the neighboring potential wells in a double-well potential (i.e., a bistable potential). The periodically switching environment plays the role of an external periodic modulation alternatively raising one potential well and lowering the other potential well. Since cellular transitions involve a sequence of coupled biochemical reactions, the process of a genetic switch is actually a continuous dynamics of a bistable process.

However, suppose that the period of the switching environment is much larger than the time scale for the cellular transition, a two-state switching model is a good approximation of the original continuous dynamics of the bistable process. Note that all the biological experiments on the microorganisms track cell populations rather than an individual cell. Assume that the cellular transition of an individual cell is independent of other cells. Hence, the two-state switching model for a large cell population is described by Eqs. (1) and (2), in which $n_{1}\left(n_{0}\right)$ represents the ensemble average of the number of cells in the fit (unfit) state. The switching rate coefficients $k_{1}$ and $k_{0}$ in 
Eqs. (1) and (2) come from the intrinsically stochastic nature of the underlying biochemical reactions. For completeness, we will explicitly write down the expressions of $k_{1}$ and $k_{0}$ in terms of the microscopic biochemical quantities. For a given genetic expression (i.e., an expressed cell state), there are many possible microscopic molecular configurations, corresponding to different combinations of regulatory proteins bound to the operator sites in the respective DNA elements. In general, the system of a particular genetic switch consists of several regulatory protein species and a specific DNA element. There are usually a few operator sites and a single promoter site in the switch DNA element. The regulatory proteins are in quasi-equilibrium with the switch DNA element. Hence, the probability of a specific molecular configuration is determined by equilibrium statistical thermodynamics. From the statistical thermodynamic theory, the switching rate coefficients $k_{1}$ and $k_{0}$ in Eqs. (1) and (2) are given as (Wolf and Arkin, 2002; Wolf and Eeckman, 1998)

$$
k_{1}=\frac{\sum_{s \in \mathrm{UNFIT}} \alpha_{s} e^{-\Delta G_{s} / R T} X_{1}^{i_{1}(s)} X_{2}^{i_{2}(s)} \cdots}{\sum_{s \in \mathrm{UNFIT}} e^{-\Delta G_{s} / R T} X_{1}^{i_{1}(s)} X_{2}^{i_{2}(s)} \cdots}
$$

and

$$
k_{0}=\frac{\sum_{s \in \mathrm{FIT}} \alpha_{s} e^{-\Delta G_{s} / R T} X_{1}^{i_{1}(s)} X_{2}^{i_{2}(s)} \cdots}{\sum_{s \in \mathrm{FIT}} e^{-\Delta G_{s} / R T} X_{1}^{i_{1}(s)} X_{2}^{i_{2}(s)} \cdots} .
$$

Here, $s$ designates the different microscopic molecular configuration and $s \in$ FIT (or UNFIT) represents all the molecular configurations which give the same genetic expression FIT (or UNFIT). $\Delta G_{s}$ is the Gibbs free energy for a specific microscopic molecular configuration $s . X_{j}$ is the concentration of the regulatory protein species $j$ and $i_{j}(s)$ is the number of regulatory proteins of the $j$ th species bound to the operator sites in the molecular configuration $s$. Other parameters include the gas constant $R=1.99 \mathrm{cal} /(\mathrm{mol} \cdot \mathrm{K})$, the absolute temperature $T$ in the Kelvin scale, and the rate coefficient $\alpha_{s}$ denoting the switching rate for a given molecular configuration $s$.

In words, a two-state switching model is a good approximation of the original continuous dynamics of the bistable process under external periodic switching environment. The intrinsically stochastic nature of biochemical reactions is expressed through the switching rate coefficients $k_{1}$ and $k_{0}$. The time average of the fraction of cells in the fit state, $f$, is the natural measure of the overall population fitness. One looks for the relations among the parameters $\left(k_{1}, k_{0}, \Delta \gamma\right)$ so that $f$, the overall population fitness, will reach optimum (or "resonance"). Thus, the problem about the long-term fitness of cells in periodically switching environment bears much resemblance to the processes of stochastic resonance. Actually, the dynamical model we study here is more complicated than the original physical processes of stochastic resonance, because cells are living things and proliferate with different rates for different cell states. Namely, this dynamical model includes the exponential growth terms and thus the cell numbers are not conserved. This is why the phase diagrams are so complicated. No simple hand waving arguments are capable of obtaining the various asymptotes. A rigorous mathematical analysis is a must do. 

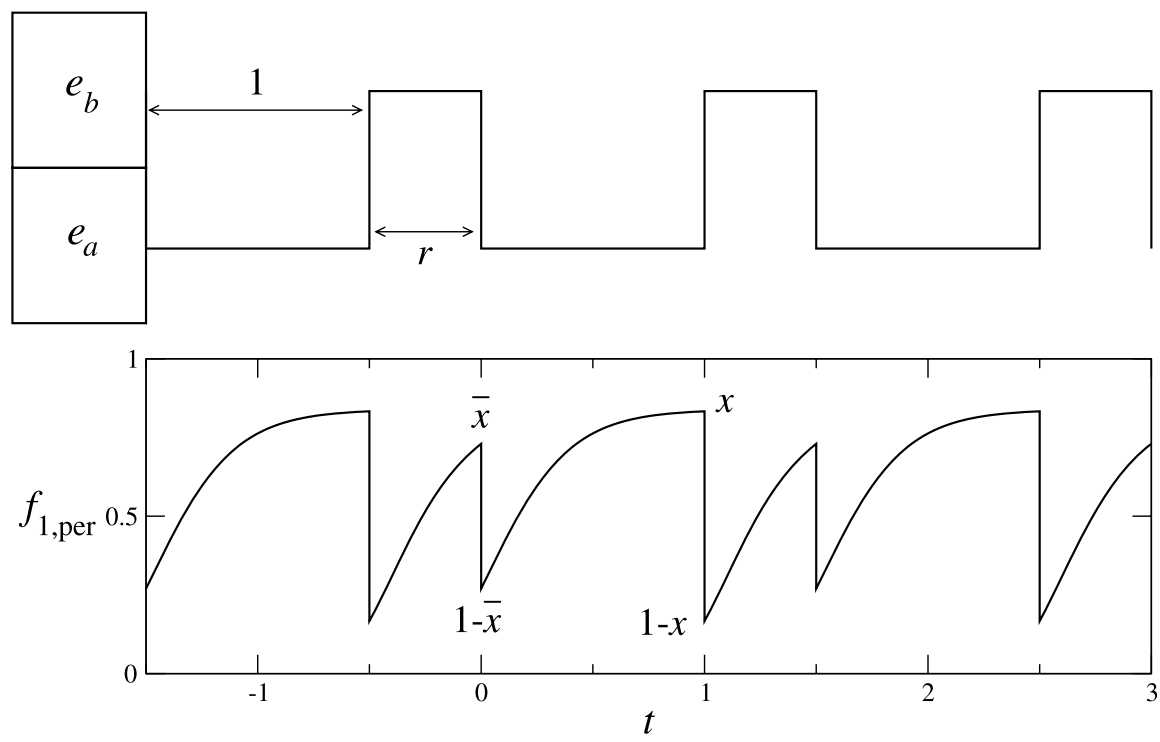

Fig. 4 The environment flips between the two states, spending times $T=1$ and $r$ in the states $e_{a}$ and $e_{b}$, respectively. We plot an illustrative figure of $f_{1, \text { per }}(t)$ vs $t$ for $r=0.5, k_{1}=1, k_{0}=1$, and $\Delta \gamma=5$. The values of $f_{1, \operatorname{per}}\left(t=[n(1+r)-r]^{-}\right)$and $f_{1, \operatorname{per}}\left(t=[n(1+r)]^{-}\right)$are denoted by $x$ and $\bar{x}$, respectively.

\section{Periodically switching environments with asymmetrical time-span}

In our preceding analysis for the cell populations under the periodically switching environments with symmetrical time-span, the time duration $T$ between the alteration of the environmental states is set to 1 . In fact, our results are applicable for any value of time duration $T$, if the values of parameters $\left(k_{1}, k_{0}, \gamma_{1}, \gamma_{0}\right)$ are rescaled by the scaling factor $T$. In the following, we would like to study the case with asymmetrical time durations between the switching of the environmental states. Without losing generality, the time durations for the environment alternately staying in the states $e_{a}$ and $e_{b}$ are set to be 1 and $r(<1)$, respectively. See Fig. 4 for illustration. In environmental state $e_{a}$, cell state $c_{a}$ is the fit state, with a similar correspondence between the environmental state $e_{b}$ and the cell state $c_{b}$. For simplicity, we assume that the switching rate coefficients $\left(k_{a}, k_{b}\right)$ and the growth rates per capita $\left(\gamma_{a}, \gamma_{b}\right)$ are symmetrical under the interchange of the two environmental states. That is, the dynamics of cell populations is still governed by Eqs. (1) and (2). Our goal is to find out what qualitative changes in the phase diagrams will arise, due to the asymmetrical time-span of the environmental states.

First, let us derive the exact solution of $f_{1, \text { per }}(t)$ in the two-state switching environment with asymmetrical time-span. By employing the relations (Eq. (5), $f_{1, \mathrm{per}}(t)=$ $f_{1, \text { per }}(t+1+r), f_{1}\left(t=[n(1+r)]^{+}\right)=1-f_{1}\left(t=[n(1+r)]^{-}\right), f_{1}\left(t=[n(1+r)-r]^{+}\right)=$ $\left.1-f_{1}\left(t=[n(1+r)-r]^{-}\right)\right)$and denoting the values of $f_{1 \text {,per }}\left(t=[n(1+r)-r]^{-}\right)$and $f_{1, \text { per }}\left(t=[n(1+r)]^{-}\right)$by $x$ and $\bar{x}$, we obtain the physically acceptable solutions of $x$ and $\bar{x}$ as

$$
x=\frac{1}{2}+\frac{1}{2}\left[\left(\rho^{r}-1\right)(\rho-1)\left(1-\tilde{f}_{+}-\tilde{f}_{-}\right)-\left(\tilde{f}_{+}-\tilde{f}_{-}\right)\left(\rho-\rho^{r}\right)\right]^{-1}
$$




$$
\begin{aligned}
& \times\left[\left(1-\tilde{f}_{+}-\tilde{f}_{-}\right)\left(\tilde{f}_{+}-\tilde{f}_{-}\right)\left(\rho^{1+r}-1\right)\right. \\
& \left.-\sqrt{\left[\left(1-\tilde{f}_{+}-\tilde{f}_{-}\right)^{2}\left(\rho^{r}-1\right)(\rho-1)+\left(\tilde{f}_{+}-\tilde{f}_{-}\right)^{2}\left(\rho+\rho^{r}\right)\right]^{2}-4\left(\tilde{f}_{+}-\tilde{f}_{-}\right)^{4} \rho^{1+r}}\right]
\end{aligned}
$$

and

$$
\bar{x}=\frac{x\left(\rho^{r}-1\right)(\rho-1)\left(1-\tilde{f}_{+}-\tilde{f}_{-}\right)+(1-x)\left(\tilde{f}_{+}-\tilde{f}_{-}\right)\left(\rho-\rho^{r}\right)}{\left(\rho^{r}-1\right)(\rho-1)\left(1-\tilde{f}_{+}-\tilde{f}_{-}\right)+\left(\tilde{f}_{+}-\tilde{f}_{-}\right)\left(\rho-\rho^{r}\right)}
$$

with $\rho \equiv \exp \left[\Delta \gamma\left(\tilde{f}_{+}-\tilde{f}_{-}\right)\right]$. See Fig. 4 for illustration. Accordingly, by employing Eq. (5) and the above properties of $f_{1, \text { per }}(t)$, we obtain the result

$$
f_{1, \operatorname{per}}(t)= \begin{cases}\tilde{f}_{+}-\frac{\tilde{f}_{+}-\tilde{f}_{-}}{1+A_{\operatorname{per}} \rho^{t-n(1+r)}}, & \text { for } n(1+r)<t<n(1+r)+1 \\ \tilde{f}_{+}-\frac{\tilde{f}_{+}-\tilde{f}_{-}}{1+\bar{A}_{\operatorname{per}} \rho^{t-n(1+r)-1}}, & \text { for } n(1+r)+1<t<(n+1)(1+r),\end{cases}
$$

with $A_{\text {per }} \equiv \frac{1-\bar{x}-\tilde{f}_{-}}{\tilde{f}_{+}-1+\bar{x}}$ and $\bar{A}_{\text {per }}=\frac{1-x-\tilde{f}_{-}}{\tilde{f}_{+}-1+x}$. Next, we will show that this periodic solution is an attractor. The time evolution of $f_{1}(t)$ can be described by the application of the following maps. The first one is the flip map $\mathcal{F}$, operating at the moment when the environmental state switches; i.e., $\mathcal{F}\left(f_{1}\right)=1-f_{1}$. The other two maps $\mathcal{C}$ and $\overline{\mathcal{C}}$ describe the changes of $f_{1}(t)$ within the environmental states $e_{a}$ and $e_{b}$, respectively, i.e.,

$$
\mathcal{C}\left(f_{1}\right)=\tilde{f}_{+}-\frac{\tilde{f}_{+}-\tilde{f}_{-}}{1+\left(\frac{f_{1}-\tilde{f}_{-}}{\tilde{f}_{+}-f_{1}}\right) \rho}
$$

and

$$
\overline{\mathcal{C}}\left(f_{1}\right)=\tilde{f}_{+}-\frac{\tilde{f}_{+}-\tilde{f}_{-}}{1+\left(\frac{f_{1}-\tilde{f}_{-}}{\tilde{f}_{+}-f_{1}}\right) \rho^{r}} .
$$

Thus, the criterion for the stability of the periodic solution is that the absolute value of $\frac{d}{d \bar{x}}[\overline{\mathcal{C}} \circ \mathcal{F} \circ \mathcal{C} \circ \mathcal{F}(\bar{x})]$ is less than 1. From $\left|\frac{d}{d \bar{x}}[\overline{\mathcal{C}} \circ \mathcal{F} \circ \mathcal{C} \circ \mathcal{F}(\bar{x})]\right|=\mid \frac{\left(x-\tilde{f}_{-}\right)\left(\tilde{f}_{+}-x\right)}{\left(1-x-\tilde{f}_{-}\right)\left(\tilde{f}_{+}-1+x\right)}$. $\frac{\left(\bar{x}-\tilde{f}_{-}\right)\left(\tilde{f}_{+}-\bar{x}\right)}{\left(1-\bar{x}-\tilde{f}_{-}\right)\left(\tilde{f}_{+}-1+\bar{x}\right)} \mid<1$, we show that the periodic solution $f_{1, \mathrm{per}}(t)$ is an attractor of $f_{1}(t)$.

After obtaining the exact solution of $f_{1, \text { per }}(t)$, the expression of the overall population fitness $f \equiv\left\langle f_{1}\right\rangle_{t}$ is obtained as

$$
\begin{aligned}
f= & \frac{\tilde{f}_{+}+\tilde{f}_{-}}{2} \\
& +\frac{1}{2(1+r) \Delta \gamma} \ln \left[\frac{\left(1-x-\tilde{f}_{-}\right)\left(\tilde{f}_{+}-1+x\right)\left(1-\bar{x}-\tilde{f}_{-}\right)\left(\tilde{f}_{+}-1+\bar{x}\right)}{\left(x-\tilde{f}_{-}\right)\left(\tilde{f}_{+}-x\right)\left(\bar{x}-\tilde{f}_{-}\right)\left(\tilde{f}_{+}-\bar{x}\right)}\right] .
\end{aligned}
$$

In the following, both analytical and numerical studies will be undertaken in order to obtain the whole picture of phase diagrams. First, we would like to analytically solve $\frac{\partial f}{\partial k_{0}}=0$ to get the relation among $k_{0, \text { opt }}$ (the optimal value of $k_{0}$ ), $k_{1}, \Delta \gamma$, and $r$. From 
Eq. (18), we have

$$
\begin{aligned}
(1+r) & \Delta \gamma\left(2 \frac{\partial f}{\partial k_{0}}+1\right) \\
= & \frac{\partial \tilde{f}_{+}}{\partial k_{0}}\left[\frac{1-2 x}{\left(\tilde{f}_{+}-1+x\right)\left(\tilde{f}_{+}-x\right)}+\frac{1-2 \bar{x}}{\left(\tilde{f}_{+}-1+\bar{x}\right)\left(\tilde{f}_{+}-\bar{x}\right)}\right] \\
& +\frac{\partial \tilde{f}_{-}}{\partial k_{0}}\left[\frac{1-2 x}{\left(1-x-\tilde{f}_{-}\right)\left(x-\tilde{f}_{-}\right)}+\frac{1-2 \bar{x}}{\left(1-\bar{x}-\tilde{f}_{-}\right)\left(\bar{x}-\tilde{f}_{-}\right)}\right] \\
& +\frac{\partial x}{\partial k_{0}}\left[\frac{2 \tilde{f}_{+}-1}{\left(\tilde{f}_{+}-1+x\right)\left(\tilde{f}_{+}-x\right)}-\frac{1-2 \tilde{f}_{-}}{\left(1-x-\tilde{f}_{-}\right)\left(x-\tilde{f}_{-}\right)}\right] \\
& +\frac{\partial \bar{x}}{\partial k_{0}}\left[\frac{2 \tilde{f}_{+}-1}{\left(\tilde{f}_{+}-1+\bar{x}\right)\left(\tilde{f}_{+}-\bar{x}\right)}-\frac{1-2 \tilde{f}_{-}}{\left(1-\bar{x}-\tilde{f}_{-}\right)\left(\bar{x}-\tilde{f}_{-}\right)}\right] .
\end{aligned}
$$

Let us express $\frac{\partial x}{\partial k_{0}}$ and $\frac{\partial \bar{x}}{\partial k_{0}}$ in terms of $\frac{\partial \tilde{f}_{+}}{\partial k_{0}}$ and $\frac{\partial \tilde{f}_{-}}{\partial k_{0}}$ :

$$
\begin{aligned}
& \left(\tilde{f}_{+}-\tilde{f}_{-}\right) \frac{\partial x}{\partial k_{0}}\left[\frac{1}{\left(1-x-\tilde{f}_{-}\right)\left(\tilde{f}_{+}-1+x\right)\left(1-\bar{x}-\tilde{f}_{-}\right)\left(\tilde{f}_{+}-1+\bar{x}\right)}\right. \\
& \left.-\frac{1}{\left(x-\tilde{f}_{-}\right)\left(\tilde{f}_{+}-x\right)\left(\bar{x}-\tilde{f}_{-}\right)\left(\tilde{f}_{+}-\bar{x}\right)}\right] \\
& =\frac{\partial \tilde{f}_{+}}{\partial k_{0}}\left[\frac{1}{\left(1-\bar{x}-\tilde{f}_{-}\right)\left(\tilde{f}_{+}-1+\bar{x}\right)}\left(\frac{1}{\tilde{f}_{+}-\bar{x}}-\frac{1}{\tilde{f}_{+}-1+x}+r \Delta \gamma\right)\right. \\
& \left.-\frac{1}{\left(\bar{x}-\tilde{f}_{-}\right)\left(\tilde{f}_{+}-\bar{x}\right)}\left(\frac{1}{\tilde{f}_{+}-x}-\frac{1}{\tilde{f}_{+}-1+\bar{x}}+\Delta \gamma\right)\right] \\
& -\frac{\partial \tilde{f}_{-}}{\partial k_{0}}\left[\frac{1}{\left(1-\bar{x}-\tilde{f}_{-}\right)\left(\tilde{f}_{+}-1+\bar{x}\right)}\left(\frac{1}{1-x-\tilde{f}_{-}}-\frac{1}{\bar{x}-\tilde{f}_{-}}+r \Delta \gamma\right)\right. \\
& \left.-\frac{1}{\left(\bar{x}-\tilde{f}_{-}\right)\left(\tilde{f}_{+}-\bar{x}\right)}\left(\frac{1}{1-\bar{x}-\tilde{f}_{-}}-\frac{1}{x-\tilde{f}_{-}}+\Delta \gamma\right)\right]
\end{aligned}
$$

and

$$
\begin{aligned}
\left(\tilde{f}_{+}\right. & \left.-\tilde{f}_{-}\right) \frac{\partial \bar{x}}{\partial k_{0}}\left[\frac{1}{\left(x-\tilde{f}_{-}\right)\left(\tilde{f}_{+}-x\right)\left(\bar{x}-\tilde{f}_{-}\right)\left(\tilde{f}_{+}-\bar{x}\right)}\right. \\
& \left.-\frac{1}{\left(1-x-\tilde{f}_{-}\right)\left(\tilde{f}_{+}-1+x\right)\left(1-\bar{x}-\tilde{f}_{-}\right)\left(\tilde{f}_{+}-1+\bar{x}\right)}\right] \\
= & \frac{\partial \tilde{f}_{+}}{\partial k_{0}}\left[\frac{1}{\left(x-\tilde{f}_{-}\right)\left(\tilde{f}_{+}-x\right)}\left(\frac{1}{\tilde{f}_{+}-\bar{x}}-\frac{1}{\tilde{f}_{+}-1+x}+r \Delta \gamma\right)\right. \\
& \left.-\frac{1}{\left(1-x-\tilde{f}_{-}\right)\left(\tilde{f}_{+}-1+x\right)}\left(\frac{1}{\tilde{f}_{+}-x}-\frac{1}{\tilde{f}_{+}-1+\bar{x}}+\Delta \gamma\right)\right]
\end{aligned}
$$




$$
\begin{aligned}
& -\frac{\partial \tilde{f}_{-}}{\partial k_{0}}\left[\frac{1}{\left(x-\tilde{f}_{-}\right)\left(\tilde{f}_{+}-x\right)}\left(\frac{1}{1-x-\tilde{f}_{-}}-\frac{1}{\bar{x}-\tilde{f}_{-}}+r \Delta \gamma\right)\right. \\
& \left.-\frac{1}{\left(1-x-\tilde{f}_{-}\right)\left(\tilde{f}_{+}-1+x\right)}\left(\frac{1}{1-\bar{x}-\tilde{f}_{-}}-\frac{1}{x-\tilde{f}_{-}}+\Delta \gamma\right)\right] .
\end{aligned}
$$

By substituting Eqs. (4), (20), and (21) into Eq. (19) and with some tedious calculation, we finally obtain the following relation among $k_{0, \text { opt }}, k_{1}, \Delta \gamma$, and $r$ :

$$
\begin{aligned}
0= & \frac{2}{1+r}(x+\bar{x}-1)\left(1+\frac{3 k_{1}}{\Delta \gamma}-\frac{k_{0, \mathrm{opt}}}{\Delta \gamma}\right)-k_{1}\left(1+\frac{k_{1}}{\Delta \gamma}-\frac{3 k_{0, \mathrm{opt}}}{\Delta \gamma}\right) \\
& -\frac{2 k_{1}}{\Delta \gamma}\left(\frac{1-r}{1+r}\right)\left(k_{1}+k_{0, \mathrm{opt}}\right)(\bar{x}-x)-\Delta \gamma\left[\frac{x(1-\bar{x})+r \bar{x}(1-x)}{1+r}\right] \\
& \times\left[1+\frac{k_{1}}{\Delta \gamma}-\frac{3 k_{0, \mathrm{opt}}}{\Delta \gamma}+2\left(\frac{k_{1}+k_{0, \mathrm{opt}}}{\Delta \gamma}\right)^{2}\right]
\end{aligned}
$$

Subsequently, we will analytically show that $k_{0, \text { opt }} \rightarrow \frac{2}{1+r}-k_{1}$ for $0 \leq k_{1} \leq \frac{2}{1+r}$ as $\Delta \gamma \rightarrow \infty$. By using $\rho^{-1}$ and $\rho^{-r}$ as small parameters for expansion, $x$ and $\bar{x}$ can be recast as

$$
x=\tilde{f}_{+}-\rho^{-1} \frac{\left(\tilde{f}_{+}-\tilde{f}_{-}\right)\left(2 \tilde{f}_{+}-1\right)}{\left(1-\tilde{f}_{+}-\tilde{f}_{-}\right)}+O\left(\rho^{-2}\right)
$$

and

$$
\bar{x}=\tilde{f}_{+}-\rho^{-r} \frac{\left(\tilde{f}_{+}-\tilde{f}_{-}\right)\left(2 \tilde{f}_{+}-1\right)}{\left(1-\tilde{f}_{+}-\tilde{f}_{-}\right)}+O\left(\rho^{-2 r}\right) .
$$

By substituting the above expressions of $x$ and $\bar{x}$ into Eq. (22) and with some tedious calculation, we get

$$
\begin{aligned}
0= & (1+r) \Delta \gamma\left(1-\frac{k_{1}}{\Delta \gamma}\right)+\frac{2 \Delta \gamma}{k_{1}+k_{0, \mathrm{opt}}} \\
& +2\left(1-\frac{2 k_{1}}{\Delta \gamma}\right)\left(1-\frac{k_{1}-k_{0, \mathrm{opt}}}{\Delta \gamma}\right)+O\left[(\Delta \gamma)^{-3}\right] .
\end{aligned}
$$

Thus, $k_{0, \text { opt }} \rightarrow \frac{2}{1+r}-k_{1}$ for $0 \leq k_{1} \leq \frac{2}{1+r}$ as $\Delta \gamma \rightarrow \infty$.

Next, by employing Eq. (22), we undertake a numerical investigation. The phase boundaries between the homogeneous phase $\left(k_{0, \text { opt }}=0\right)$ and the heterogeneous phase $\left(k_{0, \text { opt }}>0\right)$ for various values of $r$ are numerically generated. We observe that the homogeneous phase progresses through the phase space of small $\Delta \gamma$, as the value of $r$ decreases. For demonstration, Fig. 5 shows the phase boundaries between the homogeneous phase and the heterogeneous phase for the parameter $r=1,0.7$, and 0.4 , respectively. Qualitatively, this phenomenon can be understood as follows. Recall that the parameters $r$ and $\Delta \gamma$ denote the ratio of the time durations and the difference between growth rates per capita of two environmental states, respectively. If the value of $r$ is small, the time 


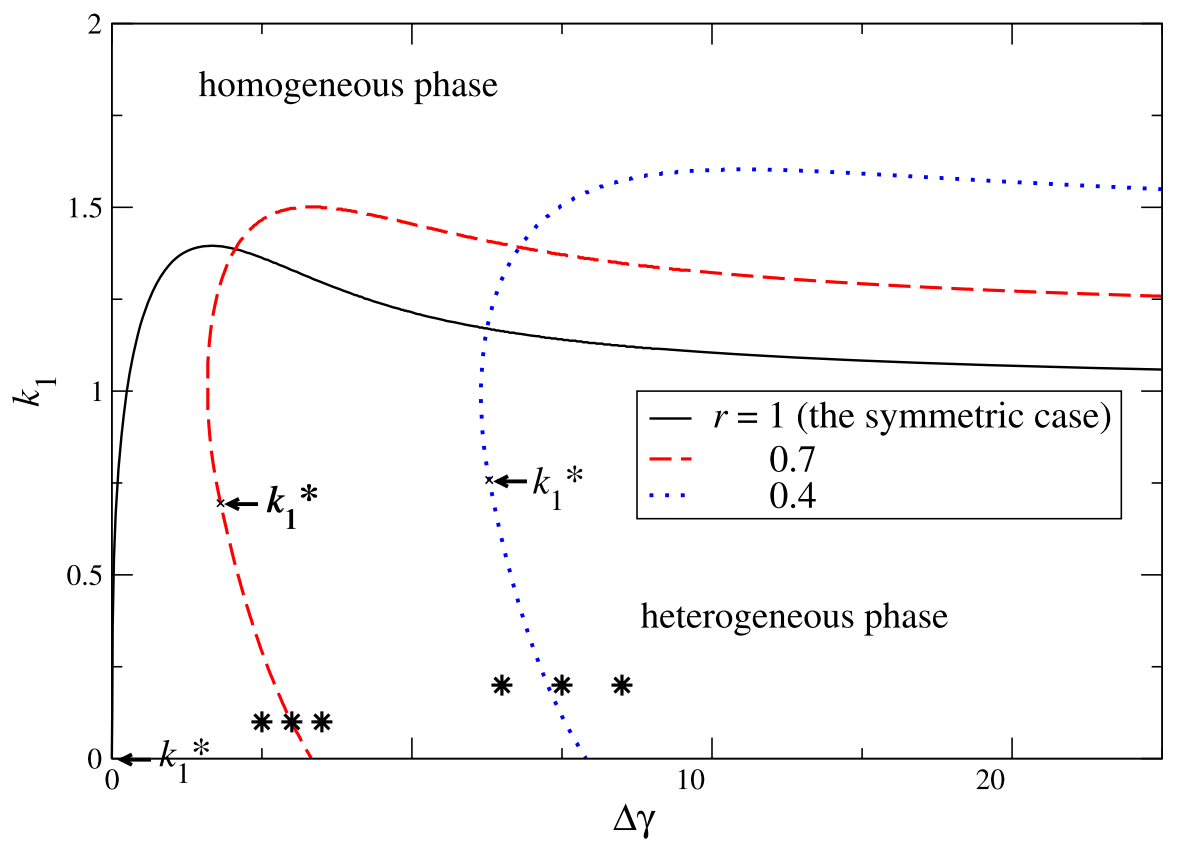

Fig. 5 The curves represent boundaries between the homogeneous phase $\left(k_{0, \mathrm{opt}}=0\right)$ and the heterogeneous phase $\left(k_{0, \text { opt }}>0\right)$ for the parameter $r=1,0.7$, and 0.4 . In each curve, the arrow symbol indicates the location of $k_{1}^{*}$. In the region with the value of $k_{1}>k_{1}^{*}$, the phase transition is continuous. In contrast, the phase transition is discontinuous in the region with $k_{1}<k_{1}^{*}$. The overall population fitness of $f$ vs $k_{0}$, with the values of $\left(k_{1}, \Delta \gamma\right)$ indicated by the star symbols adjacent to the phase boundaries, will be drawn in Fig. 6.

duration of one environmental state is much shorter than that of the other environmental state. In order to gain the overall population fitness, the cell population tends to neglect brief environmental change and only respond to the temporally dominant environmental state, if the value of $\Delta \gamma$ is small. Consequently, the heterogeneous phase recedes from the phase space of small $\Delta \gamma$, as the value of $r$ decreases.

In Figs. 6 and 7, we show the most distinct feature for the asymmetrical case: the transition from the homogeneous phase to the heterogeneous phase, with the value of $k_{1}<k_{1}^{*}$, is a discontinuous phase transition. Namely, the order parameter $k_{0 \text {,opt }}$ changes discontinuously as one crosses the phase boundary in the region with the value of $k_{1}<k_{1}^{*}$. On the other hand, the phase transition is continuous as one crosses the phase boundary in the region with $k_{1}>k_{1}^{*}$. In Fig. 5, the location of $k_{1}^{*}$ is indicated by an arrow symbol. As an illustration, we draw the overall population fitness $f$ versus $k_{0}$ in Fig. 6 with the values of $\left(k_{1}, \Delta \gamma\right)$ indicated by the star symbols in Fig. 5, adjacent to the phase boundaries. The left plot in Fig. 6 shows the fitness $f$ versus $k_{0}$ with the parameters $r=0.7, k_{1}=0.1$, and $\Delta \gamma=5,6$, and 7; the right plot in Fig. 6 shows $f$ versus $k_{0}$ with $r=0.4, k_{1}=0.2$, and $\Delta \gamma=13,15$, and 17. In Fig. 6 , the locations of $k_{0, \text { opt }}$ 's are indicated by the arrow symbols. We observe that, in the region of $k_{1}<k_{1}^{*}$, the sigmoidal shape of the function $f$ versus $k_{0}$ causes the discontinuous change of $k_{0, \text { opt }}$ from zero to a finite nonzero value. 

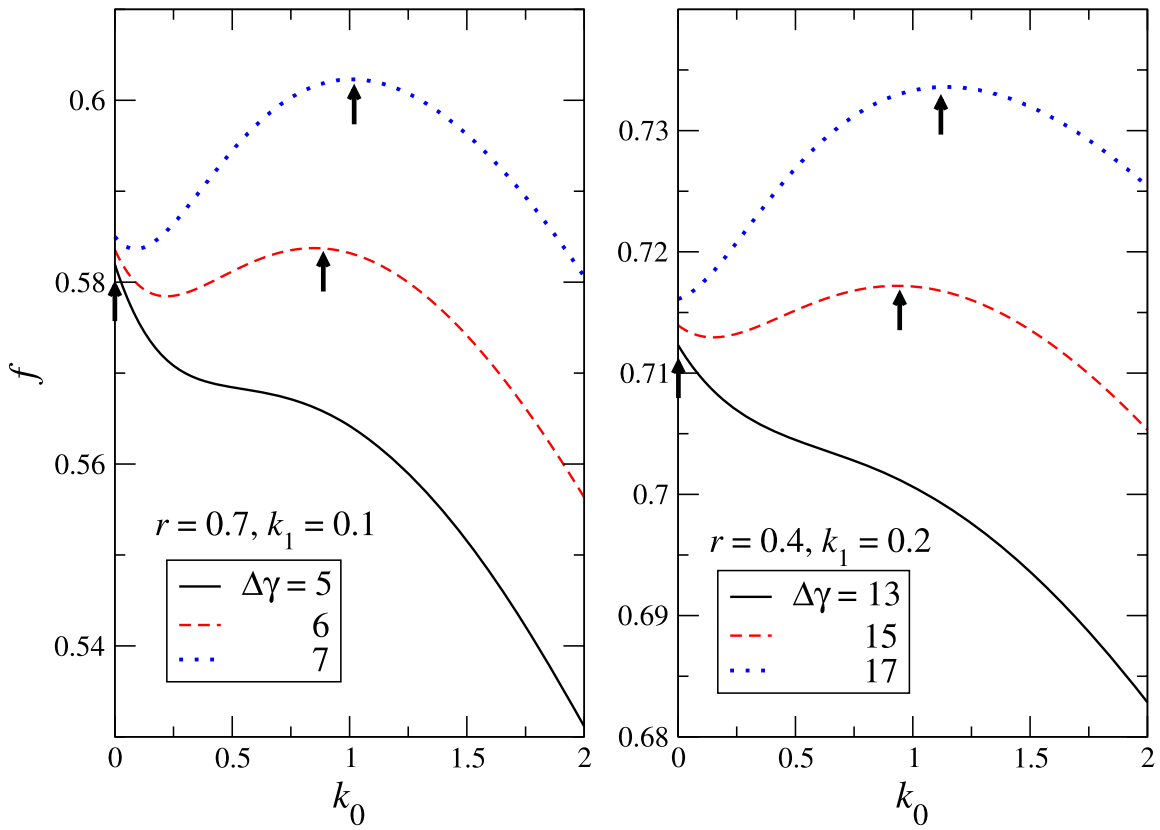

Fig. 6 Growth of cell populations in periodically switching environments with asymmetrical time-span. (Left) A plot of the overall population fitness $f$ vs $k_{0}$ with the parameters $r=0.7, k_{1}=0.1$, and $\Delta \gamma=5$, 6, and 7. (Right) A plot of the overall population fitness $f$ vs $k_{0}$ with the parameters $r=0.4, k_{1}=0.2$, and $\Delta \gamma=13,15$, and 17 . In each curve, the location of $k_{0 \text {,opt }}$ is indicated by an arrow symbol.

In addition, Fig. 7 gives a plot of $k_{0, \text { opt }}$ versus $k_{1}$ with the parameters $r=0.7$ and $\Delta \gamma=$ 5, 10, and 30. In Fig. 7, the curve of $k_{0, \text { opt }}$ versus $k_{1}$ with $\Delta \gamma=5$ clearly demonstrates the discontinuity of $k_{0, \text { opt }}$ as one crosses the phase boundary within the region of $k_{1}<k_{1}^{*}$. The solid line in Fig. 7 represents the analytically obtained asymptote: $k_{0, \mathrm{opt}} \rightarrow \frac{2}{1+r}-k_{1}$ for $0 \leq k_{1} \leq \frac{2}{1+r}$ as $\Delta \gamma \rightarrow \infty$. The curves of $k_{0, \text { opt }}$ versus $k_{1}$ with $\Delta \gamma=10$ and 30 demonstrate that the convergence of the system behaviors towards the large $\Delta \gamma$ limit is very fast. Note that, for the case with symmetrical time-span between the switching of environmental states, the transition from the homogeneous phase to the heterogeneous phase is always a continuous phase transition. Namely, the order parameter $k_{0, \text { opt }}$ always changes continuously, regardless of the values of $k_{1}$ and $\Delta \gamma$, as shown in Figs. 2 and 3. Hence, the breaking of the temporal symmetry between the switching of environmental states causes the change in the type of phase transition the system experiences.

\section{Concluding remarks}

In words, in some situations, the heterogeneous cell population may be fitter than the homogeneous cell population. This result has important biological implications. Recall that biochemical networks are performed by molecules that interact with each other. These interactions are intrinsically stochastic. Stochastic effects in gene expression account for 


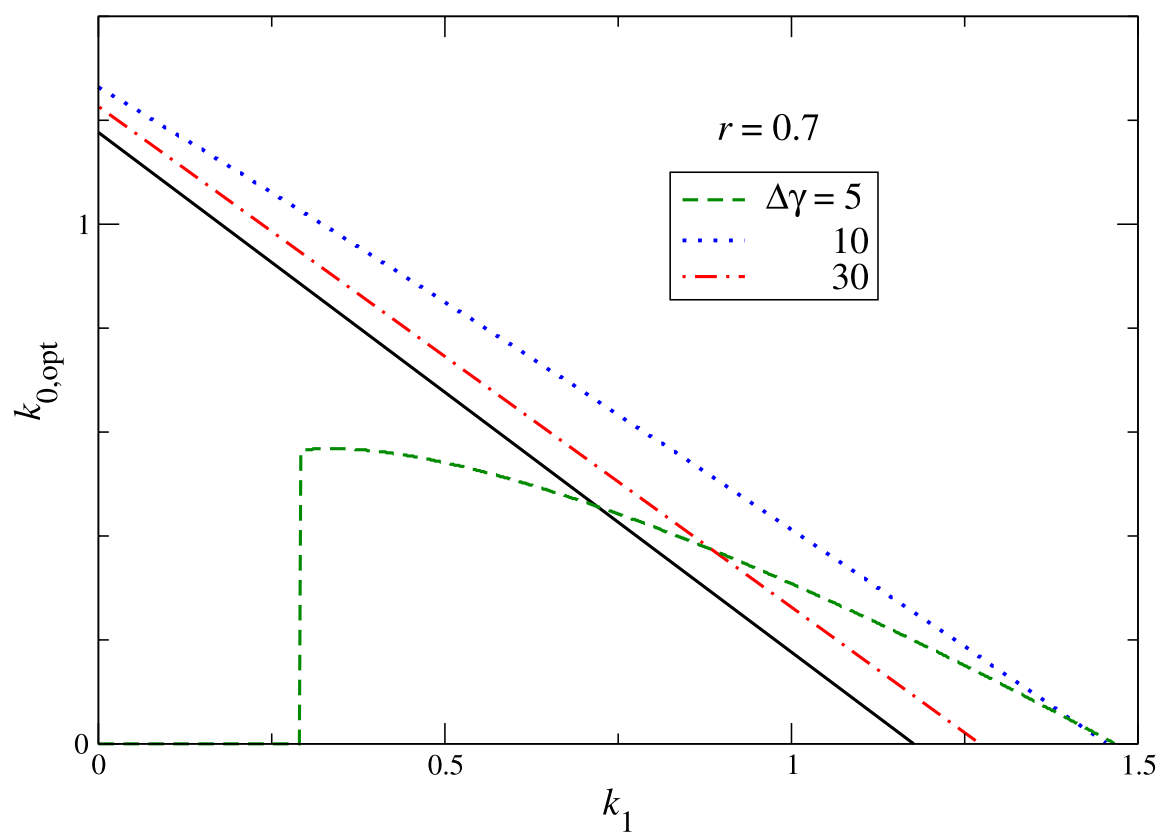

Fig. 7 A plot of $k_{0, \text { opt }}$ vs $k_{1}$ with $\Delta \gamma=5,10$, and 30, for the cell populations in periodically switching environments with asymmetrical time-span and the parameter $r=0.7$. The solid line represents the analytically obtained asymptote: $k_{0, \mathrm{opt}} \rightarrow \frac{2}{1+r}-k_{1}$ for $0 \leq k_{1} \leq \frac{2}{1+r}$ as $\Delta \gamma \rightarrow \infty$.

the cell-cell variation observed in isogenic populations (Spudich and Koshland, 1976; Paulsson, 2004; Wolf and Arkin, 2003; Kepler and Elston, 2001; Gardner et al., 2000). It is generally believed that the design of network must contain the ability of resisting biochemical noise (to avoid being error-prone) (Murray, 2002). For example, some people discuss the way to enhance the stability of genetic switches by overlapping upstream regulatory domains and arranging the adjacent operons on the DNA transcribed in diverging directions (Warren and ten Wolde, 2004). Other people discuss about how the autoregulation of biochemical networks can suppress the noise (Becskei and Serrano, 2000; Barkai and Leibler, 1997). However, in fluctuating environment, we see that phenotypic heterogeneity (induced by the intrinsic stochastic nature of biochemical reactions) can actually be a positive effect for cells to maximize their overall fitness in the long term. Moreover, in recent years, many genetic experimental results indicate the concept of adaptive mutation, whereby genetic variation apparently occurs in response to the environment (Rosenberg, 2001; Tanaka et al., 2003).

In this paper, we take extensive analytical and numerical studies on the long-term population fitness in periodically switching environment either with symmetrical timespan or asymmetrical time-span. A complete picture of phase diagrams is obtained. For the symmetrical case, the transition from the homogeneous phase to the heterogeneous phase is always a continuous phase transition. In contrast, for the asymmetrical case, the phase transition could be discontinuous in some regions of the parameter space. In addition, we also consider the switching dynamics of cell populations with the cellular 
transition time explicitly taken into account. Interestingly, we find that the systems with time-dependent cellular transitions collapse to the set of dynamical equations Eqs. (1) and (2) with the effective modified parameters. Furthermore, we point out how the problem of the overall population fitness in switching environment can be related to the renowned phenomenon, stochastic resonance, in physics and engineering. We believe that our results are helpful for the geneticists to design the artificial evolution experiments and to tackle the intriguing features of evolution and development.

\section{Acknowledgements}

The works of N.-N. Pang and W.-J. Tzeng are supported in part by the National Science Council of the Republic of China under Grant Nos. NSC-95-2112-M002-014 and NSC95-2112-M032-006, respectively.

\section{References}

Barkai, N., Leibler, S., 1997. Robustness in simple biochemical networks. Nature 387, 913-917.

Becskei, A., Serrano, L., 2000. Engineering stability in gene networks by autoregulation. Nature 405, 590-593.

Cherry, J.L., Adler, F.R., 2000. How to make a biological switch. J. Theor. Biol. 203, 117-133.

Gammaitoni, L., Hanggi, P., Jung, P., Marchesoni, F., 1998. Stochastic resonance. Rev. Mod. Phys. 70, 223-287.

Gardner, T.S., Cantor, C.R., Collins, J.J., 2000. Construction of a genetic toggle switch in Escherichia coli. Nature 403, 339-342.

Hernday, A., Krabbe, M., Braaten, B., Low, D., 2002. Self-perpetuating epigenetic pili switches in bacteria. Proc. Natl. Acad. Sci. U.S.A. 99, 16470-16476.

Kepler, T.B., Elston, T.C., 2001. Stochasticity in transcriptional regulation: origins, consequences, and mathematical representations. Biophys. J. 81, 3116-3136.

Macnab, R.M., 1992. Genetics and biogenesis of bacterial flagella. Annu. Rev. Genet. 26, 129-156.

Moss, F., 1994. Stochastic resonance: from the ice ages to the monkey's ear. In: Weiss, G.H. (Ed.), Contemporary Problems in Statistical Physics. SIAM, Philadelphia.

Murray, J.D., 2002. Mathematical Biology. Springer, Berlin.

Paulsson, J., 2004. Summing up the noise in gene networks. Nature 427, 415-418.

Rao, C.V., Wolf, D.M., Arkin, A.P., 2002. Control, exploitation and tolerance of intracellular noise. Nature 420, 231-237.

Rosenberg, S.M., 2001. Evolving responsively: adaptive mutations. Nat. Rev. Genet. 2, 504-515.

Schlax, P.J., Capp, M.W., Record, M.T. Jr., 1995. Inhibition of transcription initiation by lac repressor. J. Mol. Biol. 245, 331-350.

Spudich, J.L., Koshland, D.E., 1976. Non-genetic individuality: chance in the single cell. Nature 262, $467-471$.

Tanaka, M.M., Bergstrom, C.T., Levin, B.R., 2003. The evolution of mutator genes in bacterial populations: the role of environmental changes and timing. Genetics 164, 843-854.

Thattai, M., van Oudenaarden, A., 2004. Stochastic gene expression in fluctuating environments. Genetics $167,523-530$.

Unden, G., Becker, S., Bongaerts, J., Holighaus, G., Schirawski, J., Six, S., 1995. $\mathrm{O}_{2}$-sensing and $\mathrm{O}_{2}-$ dependent gene regulation in facultatively anaerobic bateria. Arch. Microbiol. 164, 81-90.

Vilar, J.M.G., Kueh, H.Y., Barkai, N., Leibler, S., 2002. Mechanisms of noise-resistance in genetic oscillators. Proc. Natl. Acad. Sci. U.S.A. 99, 5988-5992.

Warren, P.B., ten Wolde, P.R., 2004. Enhancement of the stability of genetic switches by overlapping upstream regulatory domains. Phys. Rev. Lett. 92, 128101- 14.

Wolf, D.M., Arkin, A.P., 2002. Fifteen minutes of fim: control of type 1 pili expression in E. coli. OMICS 6, 91-114. 
Wolf, D.M., Arkin, A.P., 2003. Motifs, modules and games in bacteria. Curr. Opin. Microbiol. 6, $125-134$.

Wolf, D.M., Eeckman, F.H., 1998. On the relationship between genomic regulatory element organization and gene regulatory dynamics. J. Theor. Biol. 195, 167-186. 